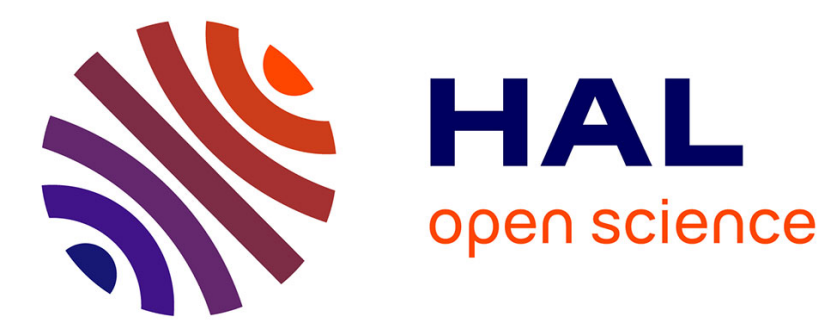

\title{
A major ice component in Pluto's haze
}

\author{
P. Lavvas, E. Lellouch, D. Strobel, M. Gurwell, A. Cheng, L. Young, G. \\ Gladstone
}

\section{To cite this version:}

P. Lavvas, E. Lellouch, D. Strobel, M. Gurwell, A. Cheng, et al.. A major ice component in Pluto's haze. Nature Astronomy, 2020, 5 (3), pp.289-297. 10.1038/s41550-020-01270-3 . hal-03418956

\section{HAL Id: hal-03418956 \\ https://hal.science/hal-03418956}

Submitted on 8 Nov 2021

HAL is a multi-disciplinary open access archive for the deposit and dissemination of scientific research documents, whether they are published or not. The documents may come from teaching and research institutions in France or abroad, or from public or private research centers.
L'archive ouverte pluridisciplinaire HAL, est destinée au dépôt et à la diffusion de documents scientifiques de niveau recherche, publiés ou non, émanant des établissements d'enseignement et de recherche français ou étrangers, des laboratoires publics ou privés. 
2

\author{
P. Lavvas ${ }^{1 *}$, E. Lellouch ${ }^{2}$, D.F. Strobel ${ }^{3}$, M.A. Gurwell ${ }^{4}$, A. Cheng ${ }^{3}$, L.A.Young 5 , R. Gladstone 6 \\ 1. GSMA UMR CNRS 7331, Université de Reims Champagne Ardenne, Reims, 51687, France \\ (panayotis.lavvas@univ-reims.fr) \\ 2. LESIA, Observatoire de Paris, PSL Research University, CNRS, Sorbonne Universités, UPMC Univ. Paris \\ 06, Univ. Paris Diderot, Sorbonne Paris Cité, 5 place Jules Janssen, 92195 Meudon, France \\ 3. Departments of Earth \& Planetary Sciences and Physics \& Astronomy, Johns Hopkins University, 3400 \\ N. Charles Street, Baltimore, MD 21218, United States \\ 4. Harvard-Smithsonian Center for Astrophysics, Cambridge, MA 02138, USA \\ 5. Southwest Research Institute, Boulder, CO 80302, USA \\ 6. Southwest Research Institute, San Antonio, TX 78238, USA
}

Pluto, Titan, and Triton, are all low-temperature environments with a $\mathrm{N}_{2} / \mathrm{CH}_{4} / \mathrm{CO}$ atmospheric composition on which solar radiation drives an intense organic photochemistry. Titan is rich in atmospheric hazes and Cassini-Huygens observations showed their formation initiates with the production of large molecules through ion-neutral reactions. New Horizons revealed that optical hazes are also ubiquitous in Pluto's atmosphere and it is thought that similar haze formation pathways are active in this atmosphere as well. However, we show here that Pluto's hazes may contain a major organic ice component (dominated by $\mathrm{C}_{4} \mathrm{H}_{2}$ ice) from the direct condensation of the primary photochemical products in this atmosphere. This contribution may imply that haze has a less important role in controlling Pluto's atmospheric thermal balance compared to Titan. Moreover, we expect that the haze composition of Triton is dominated by $\mathrm{C}_{2} \mathrm{H}_{4}$ ice.

Pluto's atmosphere is the equivalent of Titan's upper atmosphere above $400 \mathrm{~km}$ altitude, with comparable $\mathrm{CH}_{4}, \mathrm{CO}$, and $\mathrm{N}_{2}$ density profiles and pressure scale heights ${ }^{1}$. Photochemistry models for these environments demonstrate that the anticipated chemical products are similar ${ }^{2,3}$, therefore Pluto's hazes are thought to be of a similar nature based on molecular growth ${ }^{4}$ (see Methods for haze nomenclature). The fraction of the mass flux generated from the photolysis of Titan's main atmospheric composition that ends in haze particles is $\sim 30 \% 5,6$. Such a yield for Pluto's haze would suggest a mass flux of $\sim 6 \times 10^{-15} \mathrm{gcm}^{-2} \mathrm{~s}^{-1}$ (all reported mass fluxes are referred to Pluto's surface). However, the opacity of particles characterizing such a mass flux falls short of the available observations and a twice-higher haze formation efficiency is required to generate enough material to reproduce the UV extinction observations below $200 \mathrm{~km}^{7}$. As Pluto's upper atmosphere is much colder than Titan's ( $70 \mathrm{~K}$ compared to $\sim 150 \mathrm{~K}$ for Titan, see Fig. 1), an increased haze yield for Pluto is surprising. On the other hand, the photochemical gases produced on Pluto may condense at lower pressures than on Titan (Fig. 1). Therefore organic ices could be responsible for, or at least contribute to, the formation of the observed hazes in Pluto's atmosphere.

We explore the extent of this contribution using coupled models of atmospheric photochemistry and microphysics, and following the evolution of the organic ice haze particles from their formation in the upper atmosphere to their sedimentation on Pluto's surface. The models are adapted from previous studies of photochemistry and microphysics in Titan's atmosphere, taking into account high-resolution energy 
deposition, complex chemical networks, atmospheric mixing and molecular diffusion, particle sedimentation, and Brownian coagulation (see Methods).

We find that the first photochemical ice that condenses in Pluto's upper atmosphere is HCN. However, although nucleation and subsequent condensation are energetically possible, the kinetics of these processes are slow and translate into an insignificant loss for gaseous HCN above $\sim 500 \mathrm{~km}$ (Fig. 2). This result agrees with the ALMA observations of the J=4-3 line that reveal a high degree of HCN super-saturation in Pluto's upper atmosphere, near $700 \mathrm{~km}$ altitude ${ }^{8}$. We consider homogeneous nucleation, as well as nucleation in the presence of ions, with the latter being significantly more efficient than the former (see Methods). Our calculations show that at $70 \mathrm{~K}$, for $\mathrm{S}=10^{8}$ (the degree of HCN super-saturation ${ }^{8}$ near $700 \mathrm{~km}$ ) the homogeneous nucleation rate is $\sim 10^{-8} \mathrm{~cm}^{-3} \mathrm{~s}^{-1}$, that is too small for any significant condensation. For an ion density of $\sim 10^{3} \mathrm{~cm}^{-3}$, which is the peak density our calculations produce we calculate a corresponding ion nucleation rate that is more than three orders of magnitude larger than the homogeneous nucleation rate at the same conditions. Moreover, for smaller S values the ion nucleation rate drops significantly slower than the neutral nucleation rate (Extended Data Fig. 1). Heterogeneous nucleation ${ }^{9,10}$, is also a possibility if nucleation sites are present in the upper atmosphere. However, our simulated HCN profile provides a sufficiently good fit to the observed HCN line-core without it, indicating that such heterogeneous loss is relatively unimportant in Pluto's upper atmosphere.

As the HCN-ice particles settle, other gases condense and coat them. Thus, gas abundances decrease and the condensate particle radius increases (Extended Data Fig. 2). We find that a significant mass flux of the photochemical products is directly transferred to the condensed phase $\left(7.5 \times 10^{-15} \mathrm{~g} \mathrm{~cm}^{-2} \mathrm{~s}^{-1}\right)$, forming organic-ice hazes (Fig. 3a). Major contributions to particle growth come from $\mathrm{C}_{3} \mathrm{H}_{4}, \mathrm{C}_{4} \mathrm{H}_{2}, \mathrm{C}_{6} \mathrm{H}_{6}$ with massfractions varying with altitude, reflecting their individual photochemical abundances and saturation limits (Fig. 3b). In contrast, the same species in Titan's atmosphere participate in the formation of more complex gaseous compounds ${ }^{4}$, whose further chemical growth leads to the formation of Titan-type hazes ${ }^{11}$. We infer that this difference in behavior is caused by the temperature difference between Titan and Pluto. Note that condensates are also forming in Titan's atmosphere, but only in the lower stratosphere/troposphere ${ }^{12}$.

As the atmospheric density increases with decreasing altitude and particle settling velocities drop, the organic-ice hazes accumulate in the lower atmosphere. We find that below $\sim 400 \mathrm{~km}$ collisions among particles become important, leading to a transition from spherical growth, due to gas deposition, to aggregate growth, due to collisions among the formed particles (Fig. 4). At the transition region, our simulated average particle radius is $\sim 9 \mathrm{~nm}$, similar to the $10 \mathrm{~nm}$ radius derived from New Horizons observations $^{1,13}$ for the primary particles composing the haze aggregates near the surface.

Pluto's most abundant gaseous photochemical products ${ }^{14}, \mathrm{C}_{2} \mathrm{H}_{6}, \mathrm{C}_{2} \mathrm{H}_{4}$, and $\mathrm{C}_{2} \mathrm{H}_{2}$, condense only a few $\mathrm{km}$ above the surface ${ }^{15}$, and therefore should not partake in the particle formation at higher altitudes. However, the observed profiles reveal a local minimum in their abundances near $200 \mathrm{~km}$ altitude that is not reproduced by the homogeneous chemistry simulations, and instead was attributed to heterogeneous 
processes on the haze particles ${ }^{3,16}$. We evaluate the contribution of this interaction to the particle growth through an adsorption/desorption description for the $\mathrm{C}_{2}$ hydrocarbons (see Methods and Fig. 5). Our simulations suggest that heterogeneous processes mainly affect the $\mathrm{C}_{2} \mathrm{H}_{2}$ and $\mathrm{C}_{2} \mathrm{H}_{6}$ profiles, while they have smaller impact on the $\mathrm{C}_{2} \mathrm{H}_{4}$ profile. Desorption studies ${ }^{17}$ of these organic ices do demonstrate that $\mathrm{C}_{2} \mathrm{H}_{4}$ desorbs at lower temperature than $\mathrm{C}_{2} \mathrm{H}_{2}$ and $\mathrm{C}_{2} \mathrm{H}_{6}$ consistent with the qualitative picture we find here from fitting the New Horizons observations. At its maximum contribution between 100 and $200 \mathrm{~km}$ (Fig. 3), this heterogeneous coating contributes another $3.3 \times 10^{-15} \mathrm{~g} \mathrm{~cm}^{-2} \mathrm{~s}^{-1}$ to the particle mass flux. Therefore, the New Horizons observations reveal that -although not condensing- the $\mathrm{C}_{2}$-hydrocarbons do partake in the growth of the haze particles in Pluto's atmosphere. Such heterogeneous processes are also anticipated for Titan's haze ${ }^{11,18}$, but so far evidence of their role is equivocal (see Methods).

Near Pluto's temperature peak $(\sim 110 \mathrm{~K})$ between 30 and $50 \mathrm{~km}$, condensates are expected to sublimate and release their gases back to the atmosphere to an abundance limited by their individual saturation limits. Were this true, however, the HCN abundance would over-estimate the observed ALMA HCN J=4-3 satellite emissions 8 , probing altitudes near $100 \mathrm{~km}$ (blue lines, Fig. 2). Instead, we find that the heterogeneous coating is sufficiently thick to inhibit particle sublimation. Such an effect has also been observed on the Earth's secondary organic aerosol ${ }^{19}$. Considering that the effect of the coating depends on its thickness (see Methods) we derive an HCN abundance in the lower atmosphere that fits well the observed satellite emissions (red lines, Fig. 2). If the heterogeneous coating completely inhibited the sublimation of the condensed ices, we would see an even lower HCN abundance, which however does not provide a different emission spectrum (green lines, Fig. 2). Therefore, ALMA observations cannot set further constraints on the magnitude of the heterogeneous coating. However, further indications for the role of the heterogeneous component are provided by the detection of $\mathrm{CH}_{3} \mathrm{C}_{2} \mathrm{H}$ from the analysis of Pluto's UV reflectance spectrum ${ }^{20}$. Under the nominal case of partial sublimation, we calculate a $\mathrm{CH}_{3} \mathrm{C}_{2} \mathrm{H}$ column density of $2.2 \times 10^{15} \mathrm{~cm}^{-2}$, while for no sublimation we get $1.5 \times 10^{14} \mathrm{~cm}^{-2}$. The former value is closer to the observed value of $\sim 5 \times 10^{15} \mathrm{~cm}^{-2}$. We thus conclude that our simple description of the heterogeneous coating provides a representative characterization of its bulk properties.

The above processes control the variation of the average particle size and corresponding number density with altitude in Pluto's atmosphere (Extended Data Fig. 2). Note that the particle mass density is not constant with altitude, but changes reflecting the various organic ice contributions (see Extended Data Fig. 3 and Methods). Below $400 \mathrm{~km}$, organic ices provide the dominant contribution to the particle bulk mass while the heterogeneous component has a maximum contribution of $\sim 30 \%$ (Fig. 3). The optical properties of this component are unknown, and we assume here they are similar to those of the corresponding organic ices. At least for visible wavelengths where the photon penetration depths are large this assumption will have a negligible effect. The most abundant ice component is $\mathrm{C}_{4} \mathrm{H}_{2}$, thus we use it as a proxy for the evaluation of the haze optical properties. The refractive index of condensed $\mathrm{C}_{4} \mathrm{H}_{2}$ is measured in the visible ${ }^{21}$ and near IR ${ }^{22}$. In the UV, due to the lack of measurements, we derived an estimate from the gas phase component and the Kramers-Kronig relationship (see Methods and Extended Data Figs. 4 -7). 
148 Our simulated haze distribution is consistent with the New Horizons haze observations 149 at UV ${ }^{8}$, visible ${ }^{13}$ and IR $^{23}$ wavelengths (Fig. 6). We note that the UV opacity attributed to 150 hazes from the occultation measurements ${ }^{8}$ may contain contributions from unidentified gas components, and our calculations suggest that such contributions are significant above $\sim 150 \mathrm{~km}$. In addition, our simulated profiles reproduce the order of magnitude of the forward scattering and the UV extinction observations, but diverge from the observations in the back scattering near the surface, and at high altitudes $(>100 \mathrm{~km})$. These differences indicate the potential role of atmospheric circulation at high altitudes $^{24}$, the presence of horizontal haze variations ${ }^{13}$, the varying shape and optical properties of particles near the surface, as well as the limitations in the retrieval of I/F at low phase angle observations (see Methods). Notwithstanding these shortcomings, our results demonstrate that organic ices provide enough mass to reproduce observations and contribute significantly to Pluto's haze. We also present results for the case of Titantype haze formation with a mass flux of $6 \times 10^{-15} \mathrm{gcm}^{-2} \mathrm{~s}^{-1}$ assuming particles are produced in the upper atmosphere with a $10 \mathrm{~nm}$ radius and aggregate as they settle (blue lines in Fig. 6). Clearly this scenario falls short of the observations, demonstrating the need for a higher ( $\sim$ twice) haze production yield.

Nevertheless, contributions of Titan-type haze cannot be eliminated. Indeed, some of Pluto's surface features (e.g. Cthulhu region) demonstrate a spectral reddening 23,25 , similar to the red slope of Titan's aerosol analogs ${ }^{26,27}$. At Titan $11,10 \%$ of the mass flux generated by ionizing EUV radiation in the ionosphere produces the mass flux of haze embryos observed by Cassini ${ }^{28}\left(\sim 3 \times 10^{-15} \mathrm{gcm}^{-2} \mathrm{~s}^{-1}\right.$ at $\left.900 \mathrm{~km}\right)$. These embryos grow further through chemical processes before they reach the observed haze mass flux in the lower atmosphere $\left(3 \times 10^{-14} \mathrm{gcm}^{-2} \mathrm{~s}^{-1}\right)^{5}$, but the details of this chemical growth are unknown. In Pluto's ionosphere, we find that Titan-type hazes could be incepted with a mass flux of $7 \times 10^{-16} \mathrm{gcm}^{-2} \mathrm{~s}^{-1}$ based on the incoming EUV radiation. However, it is unclear how fast these embryos can chemically grow in Pluto's much colder atmosphere and in the narrow altitude range between the ionization peak $(\sim 700 \mathrm{~km})$ and the onset of condensation (below $600 \mathrm{~km}$ ). Thus, although a Titan-type haze core could exist in Pluto's haze particles, its possible significance requires further investigation.

UV and cosmic ray radiation can further modify the optical properties of organic ices towards a more absorbing signature ${ }^{29,30}$, which will make them consistent with Pluto's surface properties. We find that photons with $\lambda>150 \mathrm{~nm}$ can penetrate Pluto's lower atmosphere and reach the surface providing an energy flux of $\sim 6 \times 10^{-2} \mathrm{~W} / \mathrm{m}^{2}$ (see Methods). Due to their high energy, cosmic rays are not significantly attenuated in the atmosphere, and deposit their energy on the surface. Our simulated haze particles have an atmospheric residence time (below $200 \mathrm{~km}$ ) of about $\sim 200$ Pluto days. Given measured rates of ice modification by UV29 we estimate that particles during their settling will be affected within a depth of only $\sim 4 \mathrm{~nm}$. Moreover, the ice processing by UV radiation competes with the variable coating of the particles by the heterogeneous chemistry, which is of similar depth. Therefore, further evolution of the settling organic ice particles is more likely to occur on Pluto's surface than in the atmosphere, making their signature consistent with the observed surface reddening.

If Pluto's haze composition is dominated by organic ices, its impact on the atmospheric thermal structure will be different than that based on the currently assumed Titan-type 
composition ${ }^{31}$. Organic ices have weak absorptivity at visible and near-IR wavelengths ${ }^{32}$ that will reduce the haze heating effect, while their thermal IR opacity is limited to narrow absorption bands (compared to the continuous absorption of Titan-analogs) that will limit their cooling effect. Clearly, an updated thermal structure evaluation is required, but this task is complicated by the lack of optical properties for the organic ice mixture. A preliminary evaluation of the cooling rate based only on the narrow absorption bands of $\mathrm{C}_{4} \mathrm{H}_{2}$ ice near $40 \mu \mathrm{m}$ suggests a value of $10^{-10} \mathrm{Wm}^{-3}$ at $200 \mathrm{~km}$ that is $\sim 20$ times lower than the previous estimate based on Titan's analogs ${ }^{31}$. Clearly, a different equilibrium will be established and it remains to be evaluated if it will be able to explain Pluto's cold upper atmosphere. Another suggested coolant is water vapor resulting from the ablation of meteoroid material ${ }^{33}$. Including a source of $\mathrm{H}_{2} \mathrm{O}$ in our calculations we find that although above $\sim 400 \mathrm{~km}$ condensation is negligible, at lower altitudes the mixing ratio rapidly decreases reaching to $10^{-12}$ near $200 \mathrm{~km}$. This abundance is 10,000 times smaller than the abundance required to explain the observed temperature profile ${ }^{10}$. As other molecular candidates, (e.g. nitriles), have smaller abundances than the main species $\left(\mathrm{CH}_{4}, \mathrm{HCN}, \mathrm{C}_{2} \mathrm{H}_{2}, \mathrm{CO}\right)^{33}$ considered for the atmospheric cooling, it is potentially more promising for future studies to seek a solution not for an increased cooling contribution but rather for a reduced gas heating, i.e. a smaller atmospheric heating efficiency than the currently assumed.

Compared to Pluto, in Triton's colder atmosphere condensation could be even more important for the formed hazes ${ }^{34}$. Applying our model to Triton, we find particle formation also starts from the nucleation of HCN (Fig. 7). However, as Triton's methane is photochemically depleted at high altitudes, production of heavier hydrocarbons is prominent only at altitudes below $200 \mathrm{~km}$, where also the low temperature allows for their condensation (Fig. 7a). Thus, particle growth is efficient in the lower atmosphere with $\mathrm{C}_{2} \mathrm{H}_{4}$ providing the major contribution to the particle mass (Fig. $7 \mathrm{~b}$ ). Collisions among the formed particles become efficient below $50 \mathrm{~km}$ in Triton's atmosphere, and particles form aggregates with a primary particle radius of $\sim 20 \mathrm{~nm}$ (Fig. 7c). Heterogeneous reactions for the $\mathrm{C}_{2}$-species are less important in Triton's atmosphere because in the altitude region where they can contribute, these species also condense out; thus, we did not include them in our simulations. The resulting particle size distribution has an average bulk radius of $0.25 \mu \mathrm{m}$ near the surface with a corresponding particle density of $4 \mathrm{~cm}^{-3}$ (see Extended Data Fig. 2), while the total mass flux reaches $\sim 6 \times 10^{-15} \mathrm{gcm}^{-2} \mathrm{~s}^{-1}$. Our simulated particle distribution for Triton's organic ice haze is consistent with the Voyager 2 haze observations $35,36,37$ at multiple wavelengths/phase angles (Fig. 8), thus providing further support to our description of condensate haze formation for both Pluto and Triton.

Our work reveals two end-member processes of haze formation, i.e. molecular growth (Titan) and condensation (Triton). Pluto at the time of New Horizons observations is in between these two limits. Future observations with JWST focusing at IR wavelengths could potentially identify the spectral signatures of the main organic ices in Pluto's haze and help constrain the relative contribution of the Titan-type component. The $\mathrm{v}_{4}(3293$ $\left.\mathrm{cm}^{-1}\right)$ and $\mathrm{v}_{8}\left(664 \mathrm{~cm}^{-1}\right)$ absorption bands of solid $\mathrm{C}_{4} \mathrm{H}_{2}$ would be good starting points ${ }^{38}$, although co-condensates could demonstrate shifts relative to the anticipated monocondensate spectra ${ }^{39}$. However, given that Pluto's eccentric orbit will modify its atmospheric temperature, this variation could have an impact on the haze load, as well as on the nature of the hazes formed across the orbit. Orbiting further away from the 
245 Sun will decrease the atmospheric temperature. The main atmospheric composition is 246 likely to change with decreasing temperature, with the $\mathrm{CH}_{4} / \mathrm{CO}$ ratio decreasing with 247 increasing heliocentric distance in which case haze formation pathways based on CO 248 may become more important although with a smaller yield ${ }^{40-44}$. On the other hand the 249 reduced insolation will decrease the abundance of photochemical products. Saturation 250 vapor pressures will decrease, but the kinetics of condensation will be even slower; this 251 may reduce condensation rates, as we find here for HCN in Pluto's upper atmosphere, 252 implying that supersaturation ratios may change with distance. A self-consistent 253 evaluation of the thermal structure and composition is necessary for evaluating how the 254 haze abundance/composition varies along Pluto's orbit.

255

256

257

258

2591. Gladstone, G.R. et al. The atmosphere of Pluto as observed by New Horizons. Science 260 351, 1284-1289 (2016).

2612. Vuitton, V., Yelle, R,V., Klippenstein, S.J., Horst, S.M. \& Lavvas, P. Simulating the density of 262 organic species in the atmosphere of Titan with a coupled ion-neutral photochemical 263 model. Icarus, 324, 120-197 (2019).

2643. Wong, M.L. et al. The photochemistry of Pluto's atmosphere as illuminated by New 265 Horizons. Icarus 287, 110-115 (2017)

2664. Waite, JH,Jr., et al. The process of tholin formation in Titan's upper atmosphere. Science 267 316, 870-875 (2007)

2685. Lavvas, P., Yelle, R.V. \& Griffith C.A. Titan's vertical aerosol structure at the Huygens 269 landing site: Constraints on particle size, density, charge, and refractive index. Icarus 270 210, 832-842 (2010).

2716. Lavvas, P. et al. Energy deposition and primary chemical products in Titan's upper 272 atmosphere. Icarus 213, 233-251 (2011b)

2737. Gao, P. et al. Constraints on the microphysics of Pluto's photochemical haze from New 274 Horizons observations. Icarus 287, 116-123 (2017).

2758. Lellouch, E. et al. Detection of CO and HCN in Pluto's atmosphere with ALMA. Icarus 286, 276 289-307 (2017).

2779. Määttänen, A. et al. Nucleation studies in the Martian atmosphere. J.Geophys.Res. 110, 278 E2, E02002 (2005).

27910. Rannou, P. \& West, R.A. Supersaturation on Pluto and elsewhere. Icarus 312, 36-44 280 (2018).

28111. Lavvas, P. et al. Aerosol growth in Titan's ionosphere. PNAS 110, 2729-2734 (2013).

28212. Anderson, C.M., Samuelson, R.E. Titan's aerosol and stratospheric ice opacities between 28318 and $500 \mu \mathrm{m}$ : Vertical and spectral characteristics from Cassini CIRS. Icarus 212, 762 284778 (2011).

28513. Cheng A.F. et al. Haze in Pluto's atmosphere. Icarus 290, 112-133 (2017).

28614. Young, L.A. et al. Structure and composition of Pluto's atmosphere from the New 287 Horizons solar ultraviolet occultation. Icarus 300, 174-199 (2018).

28815. Stern S.A. et al. Evidence for possible clouds in Pluto's present-day atmosphere. Astron. J. 289 154, article id. 43 9pp (2017).

29016. Luspay-Kuti, A. et al. Photochemistry on Pluto, I. Hydrocarbons and aerosols. MNRAS 291 472, 104-117. (2017).

29217. Behmard A. et al. Desorption kinetics and binding energies of small hydrocarbons. Ap.J 293 875, article id. 73, 12pp (2019). 
29418. Lavvas, P., Sander, M., Kraft, M. \& Imanaka, H. Surface chemistry and particle shape: 295 processes for the evolution of aerosols in Titan's atmosphere. Ap.J. 728, article id. 80, 296 11pp (2011a).

29719. Vaden, T.D., Imre, D., Beránek, J., Shrivastava, M. \& Zelenyuk, A. Evaporation kinetics and 298 phase of laboratory and ambient secondary organic aerosol. PNAS 108, 2190-2195 299 (2011).

30020. Steffl, A., et al. Pluto's ultraviolet spectrum, airglow emissions, and surface reflectance. 301 EPSC-DPS2019-1213-1 (2019).

30221. Haynes, W.M. The CRC Handbook of Chemistry and Physics. Physical constants of 303 organic compounds. Taylor \& Francis Group, LLC. (2013).

30422. Khanna R.K., Ospina, M.J. \& Zhao, G. Infrared band extinctions and complex refractive 305 indices of crystalline $\mathrm{C}_{2} \mathrm{H}_{2}$ and $\mathrm{C}_{4} \mathrm{H}_{2}$. Icarus 73, 527-535 (1988).

30623. Grundy, W.M. et al. Pluto's haze as a surface material. Icarus 314, 232-245 (2018).

30724. Tanguy, B. \& Forget, F. 3D modeling of organic haze in Pluto's atmosphere. Icarus 287, 308 72-86 (2017).

30925. Protopapa, S. et al. Pluto's global surface composition through pixel-by-pixel Hapke 310 modeling of New Horizons Ralph/LEISA data. Icarus 287, 218-228 (2017).

31126. Khare B. N. et al. Optical constants of organic tholins produced in a simulated Titanian 312 atmosphere: From X-rays to microwave frequencies. Icarus 60, 127-137 (1984).

31327. Horst, S.M. Titan's atmosphere and climate. J.Geophys.Res. Planets 122, 432-482 (2017).

31428. Wahlund, J.-E. et al. On the amount of heavy molecular ions in Titan's ionosphere. Planet. 315 Space Sci. 57, 1857-1865 (2009).

31629. Gudipati, M.S., Jacovi, R., Couturier-Tamburelli, I., Lignell, A. \& Allen, M. Photochemical 317 activity of Titan's low-altitude condensed haze. Nature Communications 4, 1648-1655 318 (2013).

31930. Strazzulla, G. \& Hohnson, R.E. Irradiation effects on comets and cometary debris. In: 320 Comets in the post-Halley era, Vol I, edit: R.L. Newburn, Jr., M. Neugebauer, J Rane, 321 Astrophysics and Space Science Library, 167, Springer (1991).

32231. Zhang, X., Strobel, D.F. \& Imanaka, H. Haze heats Pluto's atmosphere yet explains its cold 323 temperature. Nature 551, 352-355 (2018).

32432. Schmitt, B., Quirico, E., Trotta, F. \& Grundy, W.M. Optical properties of ices from UV to 325 infrared, in Solar System Ices, edt. Schmitt, De Bergh, Festou. Astrophysics and Space 326 Science Library 227, Springer (1998).

32733. Strobel, D.F. \& Zhu, X. Comparative planetary nitrogen atmospheres: Density and 328 thermal structures of Pluto and Triton. Icarus 291, 55-64 (2017).

32934. Krasnopolsky, V.A., Sandel, B.R. \& Herbert, F. Properties of haze in the atmosphere of 330 Triton. J.Geophys.Res. 97, 11,695-11,700 (1992).

33135. Pollack, J.B., Schwartz, J.M. \& Rages, K. Scatterers in Triton's atmosphere: Implications 332 for the seasonal volatile cycle. Science 250, 440-443 (1990).

33336. Herbert, F. \& Sandel, B.R. $\mathrm{CH}_{4}$ and haze in Triton's lower atmosphere. J.Geophys.Res. 334 Suppl. 96, 19,241-19,252 (1991).

33537. Rages, K. \& Pollack, J.B. Voyager imaging of Triton's clouds and hazes. Icarus 99, 289-301 336 (1992).

33738. Zhou, L., Kaiser, R.I. \& Tokunaga, A.T. Infrared spectroscopy of crystalline and 338 amorphous diacetylene $\left(\mathrm{C}_{4} \mathrm{H}_{2}\right)$ and implications for Titan's atmospheric composition. 339 Planet. Space. Sci. 57, 830-835 (2009).

34039. Anderson, C.M., Samuleson, R.E. \& Nna-Mvondo, D. Organic ices in Titan's stratosphere. 341 Space Sci. Rev. 214, article id.125, 36 pp (2018).

34240. Imanaka, H., Cruikshank, D.P. \& McKay, C.P. Photochemical hazes in planetary 
343 atmospheres: solar system bodies and beyond. AAS DPS\#47, id.416.18 (2015).

34441. Hörst, S.M. et al. (2018). Haze production rates in super-Earth and mini-Neptune 345 atmosphere experiments. Nature Astronomy 2, 303-306.

34642. He, C. et al. Photochemical haze formation in the atmospheres of super-Earths and mini347 Neptunes. The Astronomical Journal 156, article id. 38, 8pp (2018).

34843. He, C. et al. Carbon monoxide affecting planetary atmospheric chemistry. The 349 Astrophysical Journal Letters 841, article id. L31, 7pp (2017).

35044. Hörst, S.M. \& Tolbert, M.A. The effect of carbon monoxide on planetary haze formation. 351 The Astrophysical Journal 781, article id. 53, 5pp, (2014).

35245. Aboudan, A., Colombatti, G., Ferri, F. \& Angrilli, F. Huygens probe entry trajectory and 353 attitude estimated simultaneously with Titan atmospheric structure by Kalman filtering.

354 Planet. Space Sci. 56, 573-585 (2008).

355

356

357

358

359

360

361

362

363

364

365

366

367

368

369

370

371

372

373

374

375

376

377

378

379

380

381

382

383

384

385

386

387

388

389

390

391
The corresponding author of this manuscript is P. Lavvas (panayotis.lavvas@univreims.fr).

Acknowledgements P.L. acknowledges support from the Programme National de Planétologie (PNP-INSU, projects: AMG \& TISSAGE).

Author Contributions P.L. designed and performed the research, and wrote the manuscript. E.L. and M.A.G. provided comparison of model results with the $A L M A$ observations. D.F.S., A.C., L.A.Y., and R.G. provided insight on the treatment of the New Horizons observations. All authors discussed the manuscript.

Materials requests \& Correspondence should be addressed to the corresponding author.

Competing interests The authors declare no competing interests. 


\section{Main Figure Legends}

Fig. 1 Thermal structures of Pluto and Titan The two black curves for Pluto present the temperature profiles derived from New Horizons ${ }^{14}$ (dashed line) and ALMA ${ }^{8}$ (solid line) observations, while the black line for Titan is the in situ profile measured by Huygens ${ }^{45}$. Altitudes for each profile are presented by the bars with associated numbers in $\mathrm{km}$. Colored lines present the saturation temperatures for different photochemical products with abundances calculated with our photochemical model. The interception point of each curve with the temperature profile (approaching from lower temperatures) indicates the approximate altitude where the condensation of each gas may start. In Pluto for example, $\mathrm{C}_{2} \mathrm{H}_{6}$ condensation is possible only near the surface, while $\mathrm{C}_{6} \mathrm{H}_{6}$ condensation starts in the upper atmosphere. In Titan all photochemical products condense in the lower $\sim 100 \mathrm{~km}$.

Fig. 2 HCN in Pluto's atmosphere Simulated HCN mixing ratio (red line) in Pluto's atmosphere relative to the anticipated saturation limit (black dashed line). The large degree of super-saturation above $\sim 250 \mathrm{~km}$ is due to condensation inefficiency owing to slow kinetics. This is consistent with the ALMA observations (central peak in inset plot, black for observed and red for the simulated emission; the gray area presents the uncertainty in the observations) ${ }^{8}$. Near $\sim 500 \mathrm{~km}, \mathrm{HCN}$ loss to condensation becomes important thus the gaseous abundance decreases. In the lower atmosphere, a saturated HCN profile is inconsistent with ALMA observations (blue line). According to our model (red line), HCN remains sub-saturated near the temperature maximum (at an altitude of $50-70 \mathrm{~km}$ ), as the coating of the particles from heterogeneous nucleation inhibits sublimation. A complete suppression of sublimation (green line) also fits the data well.

Fig. 3 Organic ice haze in Pluto's atmosphere Simulated haze mass flux in Pluto's atmosphere (panel a, black line) showing contributions of HCN nucleation (dash triple dotted), condensation of gases (dotted), and heterogeneous chemistry (dashed). The red line shows Titan's haze mass flux ${ }^{5}$ and the purple line our simulated mass flux of Triton's haze. Major contributions from the condensation of individual gases in Pluto's atmosphere, in terms of their percent contribution to the total particle mass flux, are shown on panel b with different colors and linestyles, while the short dashed black line shows the contribution of the heterogeneous component. The dash-dotted black line presents the cumulative contribution of all organic ices.

Fig. 4 Transition from spheres to aggregates in Pluto's haze Panel a presents the ratio of mass growth by coagulation to total mass growth by coagulation and condensation (the latter including heterogeneous chemistry) for different size particles at different altitudes in Pluto's atmosphere. Particles collide below $400 \mathrm{~km}$ and form aggregates. Based on these rates we derive the corresponding particle fractal dimension, $D_{f}{ }^{5}$, shown in panel b. The dashed line presents the $D_{f}$ profile used in the simulations. The inset shows that particles near the surface become more rounded due to the sharp temperature drop that allows condensation of the $\mathrm{C}_{2}$-hydrocarbons.

Fig. 5 C $_{2}$-hydrocarbons in Pluto's atmosphere Comparison of observed ${ }^{14}$ (symbols with error bars) and simulated (solid lines) line of sight densities of the $\mathrm{C}_{2}$ hydrocarbons in Pluto's atmosphere. The simulated profiles include both homogeneous and heterogeneous chemical process, with the latter considering both adsorption loss 
and desorption gain of the gases on the surface of the haze particles. The inset demonstrates how the simulated ethane profile would change if no heterogeneous loss was included (dash dotted line) or if desorption was not considered (dashed line). The

Fig. 6 Pluto's haze Comparison of simulated (black) and observed (red) haze properties for Pluto's atmosphere. Panels a-e present the scattered light observations (red lines) at different phase angles towards Pluto's North Pole at visible wavelengths ${ }^{13}$. Red crosses in panel $\mathrm{d}$ present the azimuthally averages $\mathrm{I} / \mathrm{F}$ at this phase angle, demonstrating the strong horizontal haze variations in Pluto's atmosphere. Panels $\mathrm{f}$ and g present the wavelength-averaged near-IR scattered light profile at high phase angle and the corresponding wavelength dependence of the average I/F in the lower $40 \mathrm{~km}$ of the atmosphere, respectively ${ }^{23}$. Panel $h$ presents the UV line of sight opacity ${ }^{14}$. The dashdotted line presents the cumulative opacity from organic ice haze particles (solid) and gas molecules (dashed). The blue lines present the case of Titan type haze formation with a mass flux of $6 \times 10^{-15} \mathrm{gcm}^{-2} \mathrm{~s}^{-1}$ and primary particle radius of $10 \mathrm{~nm}$. The gray shaded area presents the uncertainty in the observed opacity.

Fig. 7 Organic ice haze in Triton's atmosphere Haze mass flux (panel a) and contributions of individual gas components (panel b) for Triton's haze composition. Panels $c$ and d present the transition from spherical to aggregate growth in Triton's atmosphere. Here collisions dominate the particle growth below $50 \mathrm{~km}$.

Fig. 8 Triton's haze Simulated I/F altitude profiles of Triton's atmosphere based on our simulations of organic ice haze (lines) compared to the observed profiles (symbols) from Voyager 2 images ${ }^{35,37}$. Each panel presents observation at different spectral filters (Blue, Green, Orange, Violet at high phase angles, and Clear for low phase angle). Symbols with associated numbers correspond to the observed phase angles. For the high phase angle observations solid, dashed, and dash-dotted lines correspond to the model results for $140^{\circ}, 150^{\circ}$, and $160^{\circ}$. For the Clear filter observations the large circles and solid line present the observed and simulated I/F profiles at $60^{\circ}$ phase angle, respectively. Similarly, the small circles and dotted line present the observed ${ }^{36}$ and simulated UV tangential opacity, respectively. 


\section{Methods}

Nomenclature Through out the text the term haze is used to describe the presence of an opacity source by small particles and does not presuppose anything about their chemical composition. To make a distinction about the chemical composition of the haze particles we add the characterizations of "organic ice" haze or "Titan-type" haze, which describe hazes made through condensation of organic material or through molecular growth processes, respectively. The term tholins has a wide range of applications but here is used as a representative of the optical properties of Titan's hazes derived from laboratory experiments ${ }^{27}$. In this study we simulate exclusively the formation of organic ices, but we discuss the implications of Titan-type contributions.

Models The photochemical model is based on previous studies of Titan's photochemistry ${ }^{46}$, updated with high-resolution energy deposition calculations ${ }^{6}$ and the latest chemical reaction rates $2,47,48$. The model solves the continuity equation taking into account atmospheric mixing and molecular diffusion, the chemical production and loss processes, and loss/gain due to condensation/evaporation, for each species considered. We consider species of $\mathrm{H} / \mathrm{C} / \mathrm{N} / \mathrm{O}$ composition that can be produced from the $\mathrm{N}_{2} / \mathrm{CH}_{4} / \mathrm{CO}$ main atmospheric composition. The microphysical aspect of the simulation is based on previous models for haze and cloud formation in Titan's atmosphere ${ }^{5,49}$. These consider the Brownian (random collisions) growth of particles, as well as, their growth through the deposition of gases on their surface. The model solves for the distribution of particle sizes in a geometrically expanding grid ranging from $0.15 \mathrm{~nm}$ to $10 \mu \mathrm{m}$. The impact of atmospheric mixing and particle sedimentation (according to size and shape) on the particle distribution is included. At the top of the model (exobase) we assume escape at the Jeans escape rate for gases and no escape for haze particles, and at the bottom all simulated components (gases and hazes) are lost to the surface at the rate they arrive.

Pluto's $\mathrm{CH}_{4}$ mixing ratio at the surface is fixed at $0.4 \%$ and we assume a constant with altitude eddy mixing profile of $\mathrm{K}_{\mathrm{ZZ}}=10^{3} \mathrm{~cm}^{2} \mathrm{~s}^{-1}$. We derived the $\mathrm{K}_{\mathrm{zZ}}$ value by matching our simulated $\mathrm{CH}_{4}$ and $\mathrm{N}_{2}$ profiles in Pluto's upper atmosphere with the profiles derived from the New Horizons occultation measurements, using the temperature profile derived from that analysis ${ }^{14}$. We note, that the characteristic time scales for photochemistry/condensation or sedimentation of the main photochemical products or haze particles, respectively, are significantly smaller than the corresponding atmospheric mixing timescales. For example, $\mathrm{C}_{2} \mathrm{H}_{6}, \mathrm{C}_{2} \mathrm{H}_{4}$ and $\mathrm{C}_{2} \mathrm{H}_{2}$ have photochemical/condensation time scales at all altitudes that are smaller by factors greater than $10^{2}$ from the corresponding eddy mixing time scales. For the haze particles the sedimentation time scales are smaller from the eddy mixing time scales by factors of 5 at $10 \mathrm{~km}, 60$ at $50 \mathrm{~km}, 130$ at $100 \mathrm{~km}$, and increasing to larger values at higher altitudes. Thus, apart from altitudes near the surface, uncertainties by even a factor of 10 in the $K_{Z Z}$ will not significantly affect our conclusions.

Pluto's thermal structure exhibits a sharp temperature gradient close to the surface that requires the use of a fine grid in our diffusion calculations (Fig. 1). Thus, we use a pressure grid of 1,000 levels between the surface and Pluto's exobase that results in altitude layers of sub-km size close to the surface gradually increasing to $\sim 10 \mathrm{~km}$ at the highest altitudes of our simulation space. This altitude binning is a factor of $\sim 10$ smaller than the corresponding atmospheric scale height at each altitude.

For the energy deposition from solar photons we use high-resolution $\mathrm{N}_{2}$ cross sections at wavelengths shorter than $100 \mathrm{~nm}$, while we also consider the role of 
photoelectrons $s^{6}$ in the dissociation/ionization of $\mathrm{N}_{2}$ and $\mathrm{CH}_{4}$. We use the observed solar 540 flux on July 14th 2015 (New Horizons Pluto flyby) from the TIMED-SEE database 541 (http://lasp.colorado.edu/home/see/). At Pluto's orbit the solar insolation is small 542 enough for the contribution of resonantly scattered Ly- $\alpha$ photons from the 543 interplanetary hydrogen population (IPH) to be comparable to the direct solar 544 contribution $\left(4.7 \times 10^{8}\right.$ photons $\mathrm{cm}^{-2} \mathrm{~s}^{-1}$ at $\left.32.91 \mathrm{au}\right)$. Detailed modeling of this process 545 suggests ${ }^{50}$ that the IPH Ly- $\alpha$ background at Pluto's location at the time of the New Horizons flyby corresponds to a sky-averaged photon flux of 145 R. Thus, we consider a total Ly- $\alpha$ flux of $\sim 1 \times 10^{9}$ photons $\mathrm{cm}^{-2} \mathrm{~s}^{-1}$. We find photons dominate the $\mathrm{N}_{2}$ photolysis near $750 \mathrm{~km}$, while energetic electrons below $600 \mathrm{~km}$. $\mathrm{CH}_{4}$ photolysis peaks near 500 $\mathrm{km}$ and is dominated by Ly- $\alpha$. Pluto's ionosphere is centered near $700 \mathrm{~km}$ with a peak electron density of $\sim 10^{3} \mathrm{~cm}^{-3}$ consistent with observations ${ }^{51}$. The resulting abundance profiles of the major photochemical products demonstrate that they can condense at high altitudes in Pluto's atmosphere (Fig. 1).

For our investigation of Triton's haze we used input from the Voyager observations regarding the thermal structure 52 and energy input to the atmosphere from Neptune's magnetosphere ${ }^{52,53}$. Pluto's and Triton's atmospheres are not dense enough to attenuate galactic cosmic rays (GCR), thus we do not consider this energy source for the atmospheric chemistry. However, GCR could affect the surface optical properties (see main text and section below).

Nucleation/Condensation/Sublimation For the investigation of the HCN profile we consider neutral and ion homogeneous nucleation rates. The theory of these processes is well documented and used for various solar system studies $54,55,56$. For the evaluation of these rates we use information for the saturation vapor pressure, surface tension, mass density, and dielectric constant of condensed $\operatorname{HCN}_{55,57,58,59,60,61}$. Vapor pressures measurements at the low temperature conditions found in Pluto's and Triton's atmospheres are rare and difficult to achieve experimentally. Thus, extrapolation from higher temperature measurements is a common approach for such studies2,46,55,62,63.

New Horizons observation ${ }^{14}$ up to $350 \mathrm{~km}$ altitude indicate that haze UV extinction drops with a constant scale height of $77 \mathrm{~km}$, i.e. the haze particle size and/or density decreases with increasing altitude. Therefore, if haze particles are readily formed near Pluto's ionosphere, where they could affect the HCN condensation, their size must be small (sub-nm, based on the size of aerosols formed in Titan's ionosphere ${ }^{11}$ ), and their impact to nucleation comparable to that of the homogeneous mechanisms.

Nucleation provides the seeds on which condensation can proceed, but is only a minor loss for the gaseous abundance of HCN. As the seeds gradually increase in size through condensation, the condensation loss of gaseous HCN (as well as of other condensing species) becomes progressively more important and eventually affects the resulting gaseous abundance below $500 \mathrm{~km}$. The Kelvin effect of the saturation vapor pressure over curved surfaces is taken into account for each particle radius.

The smallest condensate particles populate the first bins of the bulk radius grid of our simulations, depending on the size they are formed through the nucleation of HCN. Subsequently, they grow to larger radii as HCN and other gases condense on their surface. We consider contributions from $\mathrm{C}_{2} \mathrm{H}_{2}, \mathrm{C}_{2} \mathrm{H}_{4}, \mathrm{C}_{2} \mathrm{H}_{6}, \mathrm{C}_{3} \mathrm{H}_{4}, \mathrm{C}_{3} \mathrm{H}_{8}, \mathrm{C}_{4} \mathrm{H}_{2}, \mathrm{C}_{4} \mathrm{H}_{8}, \mathrm{C}_{4} \mathrm{H}_{10}$, $\mathrm{C}_{6} \mathrm{H}_{2}, \mathrm{C}_{6} \mathrm{H}_{6}, \mathrm{HCN}, \mathrm{HNC}, \mathrm{CH}_{3} \mathrm{CN}, \mathrm{HC}_{3} \mathrm{~N}, \mathrm{C}_{2} \mathrm{H}_{3} \mathrm{CN}, \mathrm{C}_{2} \mathrm{H}_{5} \mathrm{CN}, \mathrm{C}_{2} \mathrm{~N}_{2}, \mathrm{C}_{4} \mathrm{~N}_{2}$, and $\mathrm{H}_{2} \mathrm{CO}$. The relative contributions from these species control the average particle mass density at each altitude, which we evaluate by calculating the mass flux of each component and the corresponding mass density of its condensate phase ${ }^{21,55}$. We had to estimate the 
densities for some of the organic ices from their liquid equivalents (species in italics above) as measurements are lacking at the required low temperatures. As demonstrated in Supplementary Table 1, typically the solid density of organic ices is higher than the corresponding liquid value with differences ranging from $\sim 4 \%$ up to $\sim 40 \%$ among the species for which both evaluations are available. For the heterogeneous component we do not know its corresponding mass density and we assume a nominal value of $1 \mathrm{gcm}^{-3}$. The simulations suggest that the particle mass density varies between $0.967 \mathrm{gcm}^{-3}$ (the mass density of HCN ice) in the upper atmosphere to an average value of $\sim 0.85 \mathrm{gcm}^{-3}$ in the lower atmosphere below $300 \mathrm{~km}$ (Extended Data Fig. 3). Assuming65 a mass density of $0.5 \mathrm{gcm}^{-3}$ for the heterogeneous component, the average particle mass density in the lower atmosphere drops to $\sim 0.75 \mathrm{gcm}^{-3}$.

The relative contributions of condensation and coagulation to the total mass growth of particles (Fig. 4 for Pluto and Fig. 7 for Triton) define the transition from spherical to aggregate growth that we describe through the aggregate fractal dimension, $D_{f}$, which varies between 3 for spheres and 2 for aggregates ${ }^{18}$.

For particle sublimation we consider the impact of the heterogeneous chemistry coating. Assuming a mass density for this component of $1 \mathrm{gcm}^{-3}$ and a typical molecular cross section of $10^{-15} \mathrm{~cm}^{2}$, the mean free path of evaporating molecules through the coating would be $\lambda_{\text {coat }} \sim 0.5 \mathrm{~nm}$. Thus, we consider that evaporation is inhibited by a factor $\exp \left(-\delta \mathrm{r} / \lambda_{\text {coat }}\right)$, where $\delta \mathrm{r}$ is the difference in the particle size (evaluated as the fractal radius ${ }^{5}$ ) due to the coating.

Heterogeneous Processes The heterogeneous loss of gases due to adsorption on particles is evaluated as $L_{H e t e r o}=\Sigma_{p}\left[n_{p} \times S_{p} \times V_{T, \text { gas }} \times n_{\text {gas }} \times f_{\text {Stick }}(T)\right]$, where, the summation is over the particle size distribution, $n_{p}$ and $S_{p}$ are the density and surface area of size-p particles, $\mathrm{V}_{\mathrm{T}, \text { gas }}$ and $\mathrm{n}_{\text {gas }}$ are the thermal velocity and density of the hitting molecules, and $\mathrm{f}_{\text {Stick }}(\mathrm{T})$ is temperature-dependent sticking efficiency of each heterogeneously lost molecule with the form $\mathrm{f}_{\text {Stick }}(\mathrm{T})=\mathrm{A}^{*}\left(1-\tanh \left(0.2^{*}(\mathrm{~T}-\mathrm{Tst})\right)\right) / 2$ with $\mathrm{A}$ and Tst gasdependent parameters. This expression, based on measured sticking efficiencies of small molecules on water ice ${ }^{64}$, describes a sigmoidal increase of sticking efficiency below temperature Tst, with a maximum sticking efficiency reaching to value A. Heterogeneous gas production due to desorption is evaluated as $P_{\text {Hetero }}=\Sigma_{p}\left[n_{p} \times S_{p} \times V_{T, a t m} \times n_{a t m} \times e_{\text {gas }} \times\right.$ $\left.\mathrm{f}_{\text {Disorpt }}(\mathrm{T})\right]$. In this expression $\mathrm{e}_{\text {gas }}$ is the contribution of each heterogeneously lost molecule to the total mass of each particle (as in Fig. 2 ) and $f_{\text {Disorpt }}=B\left(1-f_{\text {Stick }} / A\right)$. With this temperature dependence desorption is active only when sticking is inefficient due to the increased atmospheric temperature, i.e. temperature controls the interaction between gases and particles. To match the observed $\mathrm{C}_{2}$-hydrocarbon profiles (Fig. 5) with this scheme we use A values of $5 \times 10^{-6}, 1 \times 10^{-5}, 5 \times 10^{-6}$, with Tst $=82 \mathrm{~K}, 90 \mathrm{~K}, 82 \mathrm{~K}$ and $\mathrm{B}=1 \times 10^{-12}, 1 \times 10^{-12}, 3 \times 10^{-12}$ for $\mathrm{C}_{2} \mathrm{H}_{6}, \mathrm{C}_{2} \mathrm{H}_{4}$ and $\mathrm{C}_{2} \mathrm{H}_{2}$, respectively. It is also interesting to note that experiments on water ice suggest that $\mathrm{CO}, \mathrm{CH}_{4}, \mathrm{~N}_{2}$ i.e. Pluto's main atmospheric compounds, can stick on the ice surface at temperature similar to Pluto's ${ }^{64}$. However, as the nature of the substrate has a major role for the interaction with the impinging molecules, we did not consider this effect in our study. It would be useful to explore this option first through laboratory experiments on organic ices.

Various studies have suggested that there may be evidence for heterogeneous reactions in Titan's atmosphere but the results seem to be contradictory with the inclusion of heterogeneous processes improving the fits to some photochemical products and worsening them for others ${ }^{2}$. Unfortunately, the evaluation of heterogeneous reactions in Titan's atmosphere is based on short-lived photochemical 
products with most of them being significantly down the pathway of molecular growth

638 from the initial $\mathrm{N}_{2} / \mathrm{CH}_{4}$ mixture, where reaction pathways and rates are not as well known as for the first steps. On the contrary, for Pluto's atmosphere the evidence of heterogeneous processes is based on ethane and acetylene, which are long-lived photochemical products with a relatively well understood photochemistry.

Other processes The differences between observed and simulated haze properties indicate that additional processes could affect the particle properties. General circulation models ${ }^{24}$ demonstrate that for the calculated primary particle radius circulation will result in longer residence times than those calculated with our 1D simulations. This effect would increase the altitude slope in the I/F and UV extinction profiles towards the observed. Moreover, observations demonstrate a strong increase in haze scattering towards Pluto's North pole ${ }^{13}$. From high phase angle observations, the local increase near the pole can be up to a factor of three from the azimuthally average I/F (see Fig. 6). We also need to keep in mind that the UV opacity attributed to hazes in the New Horizons occultation analysis could include contributions from gaseous components not identifiable in the spectra. Near the surface, observations suggest that the particle's shape becomes more spherical relative to the aggregate shape at $50 \mathrm{~km}$. Our calculations suggest that near the surface condensation of the $\mathrm{C}_{2}$-hydrocarbons provides enough mass to the particles to affect their shape (see inset in Fig. 4). However, we did not include this effect in our current calculations. We note that changing the particle shape towards a sphere will increase the sedimentation velocity, thus rapidly decrease the local particle density. Also we did not consider the possible trapping of other gases (e.g. $\mathrm{N}_{2}, \mathrm{CH}_{4}, \mathrm{CO}$ ) in the condensates that would result in a smaller mass density, while we had to make an assumption for the mass density of the heterogeneous component $\left(1 \mathrm{~g} \mathrm{~cm}^{-3}\right)$. Changes in the particle mass density will directly affect the sedimentation velocity of the haze particles and affect their vertical profiles. Sticking of $\mathrm{N}_{2}, \mathrm{CH}_{4}$, and $\mathrm{CO}$ at the very low temperatures near the surface is also a possibility, given the efficiency of such processes on water ice ${ }^{64}$. This could further affect the particle shape and refractive index, therefore the local scattered light properties. All the above need to be evaluated in future investigations once a better understanding of the heterogeneous interaction mechanisms is achieved. Finally, note that for the low phase angle observations $\left(20^{\circ}, 67^{\circ}\right)$ the derived scattered light profiles are based on the removal of a surface scattering contribution, the latter being inferred from the surface of Charon ${ }^{13}$. The uncertainties from this approach are not well constrained.

Particle treatment by UV and cosmic rays Due to the low atmospheric density and the loss of photochemical products from condensation, as well as, the low opacity of the haze, the atmosphere beyond $\sim 150 \mathrm{~nm}$ is practically transparent and solar insolation reaches the surface. Our calculations provide an energy flux of $\sim 6 \times 10^{-6} \mathrm{Wcm}^{-2}$ in the wavelength range $200-350 \mathrm{~nm}$. Laboratory experiments ${ }^{29}$ studying the irradiation of $\mathrm{C}_{4} \mathrm{~N}_{2}$ ice at similar wavelengths show that $13 \%$ of ice thickness $(200 \mathrm{~nm})$ is polymerized when irradiated by UV light for $84,000 \mathrm{~s}$ and a flux of $0.05 \mathrm{~W} \mathrm{~cm}^{-2}$. Using this estimate for Pluto's case and considering the particle residence time in the lower $200 \mathrm{~km}\left(\sim 10^{8} \mathrm{~s}\right)$ we find that particles should be affected to a depth of $3.7 \mathrm{~nm}$. However the production of polymer material acts as a screen that absorbs the incoming high-energy photons and eventually limits the growth of the polymer. Further input from laboratory studies is required to investigate this effect on the organic ices identified here for the composition of Pluto's haze. Cosmic rays provide a higher energy input (MeV-GeV) but with lower 
686 flux. Each energetic particle can produce a cascade of high-energy photons/electrons 687 that amplifies the effect of each original impact. Cosmic rays have a higher penetration 688 depth compared to UV photons and can excite simultaneously multiple molecules in the

Refractive indices For $\mathrm{C}_{4} \mathrm{H}_{2}$ and $\mathrm{C}_{2} \mathrm{H}_{4}$ ices, which we find to be the major mass contributors for Pluto's and Triton's hazes respectively, information for their $(\mathrm{n}, \mathrm{k})$ is limited to IR wavelengths where their absorption bands are detected ${ }^{22,67}$. Moreover, the corresponding $\mathrm{n}$ values at visible wavelengths ( $\mathrm{n}_{\mathrm{VIS}}$ ) are $\mathrm{known}^{67}$, or can be estimated from the liquid phase ${ }^{21}$. It is well established that organic ices have extremely low absorptivity at visible wavelengths ${ }^{32}$, thus the lack of the exact knowledge of the kVIs values, will not affect our evaluation of the scattering properties of the particles at visible wavelengths. However, for comparison with the UV observations we do need an estimate for their $(\mathrm{n}, \mathrm{k})_{\mathrm{uv}}$. We generate such estimates based on the available refractive indices at IR wavelengths and the Kramers-Kronig closure relationships ${ }^{68}$. This is a wellestablished technique used in laboratory studies to derive the refractive index at IR based on absorptivity measurements and the known refractive index at visible, $\mathrm{n}_{\mathrm{VIS}}{ }^{22,67,69}$. Here we use this technique in a reverse approach, as the value of $\mathrm{n}_{\mathrm{VIS}}$ is dominated by the integrated contribution from shorter wavelengths. For the $\lambda$ dependence of $\mathrm{k}_{\mathrm{UV}}$ we can use the photoabsorption cross section ( $\sigma_{\mathrm{UV}}$ ) of the corresponding gas phase to derive an estimate ${ }^{70}$ for $\mathrm{k}_{U V}=\rho_{\mathrm{S}} \sigma_{U V} \lambda \mathrm{N}_{\mathrm{A}} / 4 \pi \mathrm{M}$, where $\mathrm{M}$ is the molecular mass of the molecule and $\rho_{s}$ the solid's mass density, and $\mathrm{N}_{\mathrm{A}}$ Avogadro's number. This approach has been used before for estimating the absorptivity of $\mathrm{CH}_{4}$ ice ${ }^{71}$. The accuracy of this approximation depends on the molecule considered. Studies of UV absorptivities of molecules at gas and solid phases demonstrate that the phase transition results in blue shifts of major electronic bands and the suppresion of vibrational futures ${ }^{72}$. Moreover, such effects appear to be enhanced in polar relative to non-polar molecules.

As a demonstration of our approach we present the cases of $\mathrm{CH}_{4}$ and $\mathrm{H}_{2} \mathrm{O}$ ices for which UV measurements are available ${ }^{73,74,75}$. For $\mathrm{CH}_{4}$ ice (Extended Data Figure 4), the gas-phase estimate is consistent with the measured $\mathrm{k}_{\mathrm{UV}}$ for which the laboratory measurements show that the blue shift is $\operatorname{small}^{73}(\sim 3.8 \mathrm{~nm}$ at the peak of the lowest absorption band, corresponding to energy shift of $\left.\sim 2,000 \mathrm{~cm}^{-1}\right)$. Moreover our estimate is consistent with a previous estimate based on the same approach ${ }^{71}$. For the $\mathrm{H}_{2} \mathrm{O}$ ice (Extended Data Figure 5), the gas phase estimate does not provide an as good match to the $\mathrm{n}_{\mathrm{VIS}}$ as for the $\mathrm{CH}_{4}$ case. For this polar molecule laboratory studies ${ }^{76}$ show that the blue shift is significantly larger $(\sim 23 \mathrm{~nm})$. To evaluate this effect on our calculation we fitted the $\mathrm{k}_{U V}$ from the gas phase with a set of gaussians which we subsequntly blue shifted by a constant energy. Applying a shift of $\sim 10,000 \mathrm{~cm}^{-1}$ brings the $\mathrm{k}_{\mathrm{Uv}}$ closer to the observed spectrum while also impoving the derived $n_{\text {VIS }}$ value from 1.270 to 1.294 (note that $\mathrm{n}_{\mathrm{VIS}}$ values are measured to an accuracy of $1 \%{ }^{76}$, thus this difference could be significant).

With this approach we derived the estimates for the $\mathrm{k}_{U v}$ of $\mathrm{C}_{4} \mathrm{H}_{2}$ and $\mathrm{C}_{2} \mathrm{H}_{4}$ (Extended Data Figures 6 \& 7). As these are also non-polar molecules we evaluated how the refractive index would change assuming relatively small blueshifts $\left(1,000-5,000 \mathrm{~cm}^{-1}\right.$ based on the above discussion). For the wavelength range sensitive to the haze retrieval by New Horizons $(175-188 \mathrm{~nm})$ we estimate that the average $\mathrm{C}_{4} \mathrm{H}_{2}$ ice $\mathrm{k}_{\mathrm{Uv}}$ may vary between $6 \times 10^{-3}$ and $1.5 \times 10^{-2}$. The corresponding $\mathrm{k}_{\mathrm{Uv}}$ range for $\mathrm{C}_{2} \mathrm{H}_{4}$ ice for Voyager observations $(150-160 \mathrm{~nm})$ is $0.65-0.72$. We emphasize here that these are bulk 
735 estimates used to derive an order of magnitude evaluation for the possible range of UV

736 extinction for our simulated haze distributions. Actual measurements are required for a

737 better evaluation, and we hope this work will motivate such laboratory studies.

738

739

740

Methods References

741

74246. Lavvas P.P., Coustenis A. \& Vardavas I.M. Coupling photochemistry with haze formation 743 in Titan's atmosphere, part II: Results and validation with Cassini/Huygens data. Planet 744 Space Sci. 56, 67-99 (2008).

74547. Douglas, K. et al. Low temperature studies of the removal reactions of ${ }^{1} \mathrm{CH}_{2}$ with 746 particular relevance to the atmosphere of Titan. Icarus 303, 10-21 (2018).

74748. Douglas, K. et al. Low temperature studies of the rate coefficients and branching ratios of 748 reactive loss vs quenching for the reactions of ${ }^{1} \mathrm{CH}_{2}$ with $\mathrm{C}_{2} \mathrm{H}_{6}, \mathrm{C}_{2} \mathrm{H}_{4}, \mathrm{C}_{2} \mathrm{H}_{2}$. Icarus 321, 749 752-766 (2019).

75049. Lavvas, P., Griffith, C.A. \& Yelle, R.V. Condensation in Titan's atmosphere at the Huygens 751 landing site. Icarus 215, 732-750 (2011c).

75250. Gladstone, G.R., Pryor, W.R. \& Stern, S.A. Lya@Pluto. Icarus 246, 279-284 (2015).

75351. Hinson, D.P. et al. An upper limit on Pluto's ionosphere from radio occultation

754 measurements with New Horizons. Icarus 307, 17-24 (2018).

75552. Strobel, D.F. \& Zhu, X. Comparative planetary nitrogen atmospheres: Density and 756 thermal structures of Pluto and Triton. Icarus 291, 55-64, (2017).

75753. Strobel, D.F. \& Summers, M.E. Triton's upper atmosphere and ionosphere, in Neptune 758 and Triton, edited by DP Cruikshank, University of Arizona Press (1995).

75954. Pruppacher, H.R. \& Klett, J.D. Microphysics of clouds and precipitation. Kluwer Academic 760 Press (1997).

76155. Moses, J.I., Allen, M. \& Yung, Y.L. Hydrocarbon nucleation and aerosol formation in 762 Neptune's atmosphere. Icarus 99, 318-346 (1992).

76356. Fisenko, S.P., Kane, D.B. \& El-Shall, M.S. Kinetics of ion-induced nucleation in a vapor-gas 764 mixture. Journal of Chemical Physics 123 article id.10, 104704 (2005).

76557. Fray, N. \& Schmitt, B. Sublimation of ices of astrophysical interest: A bibliographic 766 review. Planet. Space Sci. 57, 2053-2080 (2009).

76758. Dykyi, J., et al. Vapor pressure of chemicals. In: Martienssen, W. (Ed.), Landolt-Bornstein, 768 Numerical Data and Functional Relationships in Science and Technology, Group IV:

769 Physical Chemistry, vol. 20, Springer (1999).

77059. Wohlfarth, Ch. \& Wohlfarth, B. Surface tension of pure liquids and binary liquid 771 mixtures. In: Martienssen, W. (Ed.), Landolt-Bornstein, Numerical Data and Functional 772 Relationships in Science and Technology, Group IV: Physical Chemistry, vol. 16. Springer 773 (1997).

77460. Guez, L., Bruston, P., Raulin, F. \& Regnaut, C. Importance of phase changes in Titan's 775 lower atmosphere. Tools for the study of nucleation. Planet. Space Sci. 45, 611-625 776 (1997).

77761. Krause, P.F. \& Friedrich, H.B. Infrared spectra and dielectric properties of crystalline 778 hydrogen cyanide. J.Phys.Chem 76, 1140-1146 (1972).

77962. Krasnopolsky, V. Titan's photochemical model: Further update, oxygen species, and 780 comparison with Triton and Pluto. Planet Space Sci., 73, 318-326 (2012).

78163. Willacy, K., Allen, M. \& Yung, Y.L. A new astrobiological model of the atmosphere of 782 Titan. Ap.J. 829, article id. 79, 11p (2016).

78364. He J., Acharyya K. \& Gianfranco V. Sticking of molecules on nonporous amorphous water 
784 ice. Ap.J. 823, article id. 56, 10pp (2016).

78565. Hörst, S.M. \& Tolbert, M.A. In situ measurements of the size and density of Titan aerosol 786 analogs. Ap. J. Lett. 770, article i.d. L10, 6pp (2013).

78766. Henderson, B. \& Gudipati, M.S. Direct detection of complex organic products in 788 ultraviolet (Ly-a) and electron-irradiated astrophysical and cometary ice analogs using 789 two step laser ablation and ionization mass spectrometry. Ap.J. 800, article id. 66, 17pp 790 (2015).

79167. Hudson, R.L., Gerakines, P.A. \& Moore, M.H. Infrared spectra and optical constants of 792 astronomical ices: II. Ethane and ethylene. Icarus 243, 148-157 (2014).

79368. Lucarini, V., Saarinen, J.J., Peiponen, K.-E. \& Vartiainen, E.M. Kramers-Kroning relations 794 in optical material research. ISBN-13978-3-540-23673 (Springer, 2005).

79569. Masterson, C.M. \& Khanna, R.K. Absorption intensities and complex refractive indices of 796 crystalline $\mathrm{HCN}, \mathrm{HC}_{3} \mathrm{~N}$, and $\mathrm{C}_{4} \mathrm{~N}_{2}$ in the infrared region. Icarus 83, 83-92 (1990).

79770. Beyer, K.D. \& Ebeling, D.D. UV refractive indices of aqueous ammonium sulfate solutions. 798 GRL 25, 3147-3150 (1998).

79971. Martonchik, J.V. \& Orton, G.S. Optical constants of liquid and solid methane. Applied 800 Optics 33, 8306-8317 (1994).

80172. Mason, N.J. et al. VUV spectroscopy and photo-processing of astrochemical ices: an 802 experimental study. Faraday Discussions 133, 311-329 (2006).

80373. Wu, Y.-J. et al. Spectra and photolysis of pure nitrogen and methane dispersed in solid 804 nitrogen with vacuum-ultraviolet light. Ap.J. 746, article id.175, 11pp (2012).

80574. Cruz-Diaz, G.A., Muñoz Caro, G.M., Chen, Y.-J. \& Yih, T.-S. Vacuum-UV spectroscopy of 806 interstellar ice analogs. I. Absorption cross-sections of polar-ice molecules. A\&A 562, 807 A119-10 (2014).

80875. Cruz-Diaz, G.A., Muñoz Caro, G.M., Chen, Y.-J. \& Yih, T.-S. Vacuum-UV spectroscopy of 809 interstellar ice analogs. II. Absorption cross-sections of nonpolar ice molecules. $A \& A$ 810 562, A120-9 (2014).

81176. Warren, S.G. \& Brandt, R.E. Optical constants of ice from ultraviolet to the microwave: A 812 revised compilation. JGR 113, D14220-10 (2008).

81377. Grundy, W.M., Schmitt, B. \& Quirico, E. The temperature-dependent spectrum of 814 methane ice I between 0.7 and $5 \mu \mathrm{m}$ and opportunities for near-infrared remote 815 thermometry. Icarus 155, 486-496 (2002).

81678. Trotta, F. \& Schmitt, B. Determination of the optical constants of ices in the mid-infrared. 817 In The cosmic dust connection, 179-184, ed. J.M. Greenberg, Kluwer Academic 818 Publishers (1996).

81979. Protopapa, S., Grundy, W.M., Tegler, S.C. \& Bergonio, J.M. Absorption coefficients of 820 methane-nitrogen binary ice system: Implications for Pluto. Icarus 253, 179-188 (2015). 82180. Koski, H.K. \& Sandor, E. Neutron powder diffraction study of the low-temperature phase 822 of solid acetylene-d $\mathrm{d}_{2}$. Acta Cryst. B31, 350-353 (1975).

82381. Stewart, J.W. \& La Rock, R.I. Compression and densities of four solidified hydrocarbons 824 and carbon tetrafluoride at 77 oK. The Journal of Chemical Physics 28, 425 - 427 (1958).

82582. Acree, W.E. Jr. \& Chickos, J.S. "Phase Transition Enthalpy Measurements of Organic and 826 Organometallic Compounds" in NIST Chemistry WebBook, NIST Standard Reference 827 Database Number 69, Eds. P.J. Linstrom and W.G. Mallard, National Institute of 828 Standards and Technology, Gaithersburg MD, 20899, https://doi.org/10.18434/T4D303 82983. Cordier, D. et al. Structure of Titan's evaporates. Icarus 270, 41-56 (2016).

83084. Dulmage, W.J. \& Lipscomb, W.N. The crystal structures of hydrogen cyanide, HCN. Acta 831 Cryst. 4, 330-334 (1951).

83285. http://www.molbase.com/moldata/2253950.html 
83386. Shallcross, F.V. \& Carpenter, G.B. The crystal structure of cyanoacetylene. Acta Cryst. 11, 834 490-496 (1958).

835

836 Data availability The data that support the plots within this paper and other findings of 837 this study are available from the corresponding author upon reasonable request.

838

839 Code availability The codes used in this study are described in detail in previous 840 relevant publications (see references). They are not publicly available owing to their 841 undocumented intricacies.

842

843

844 


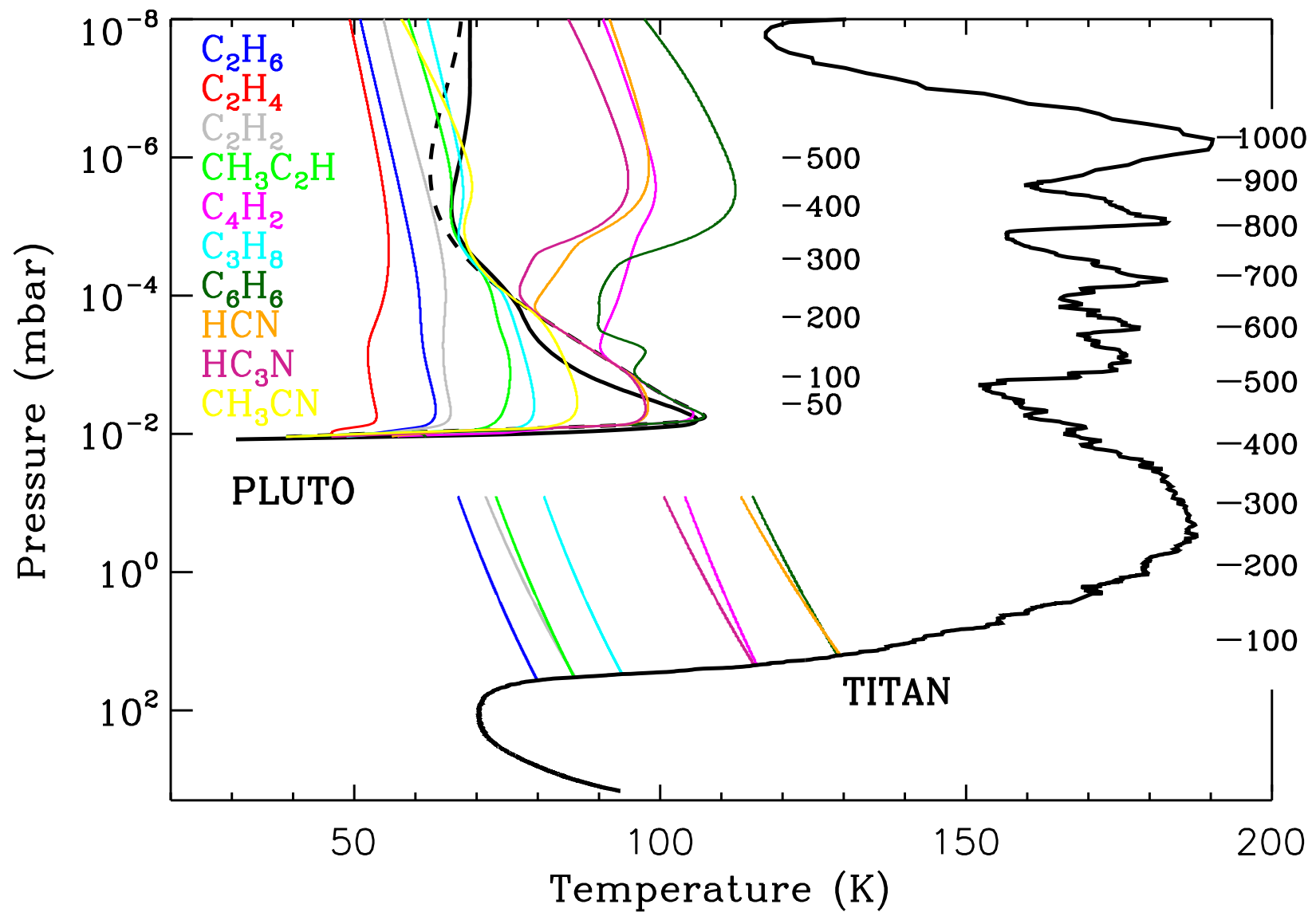




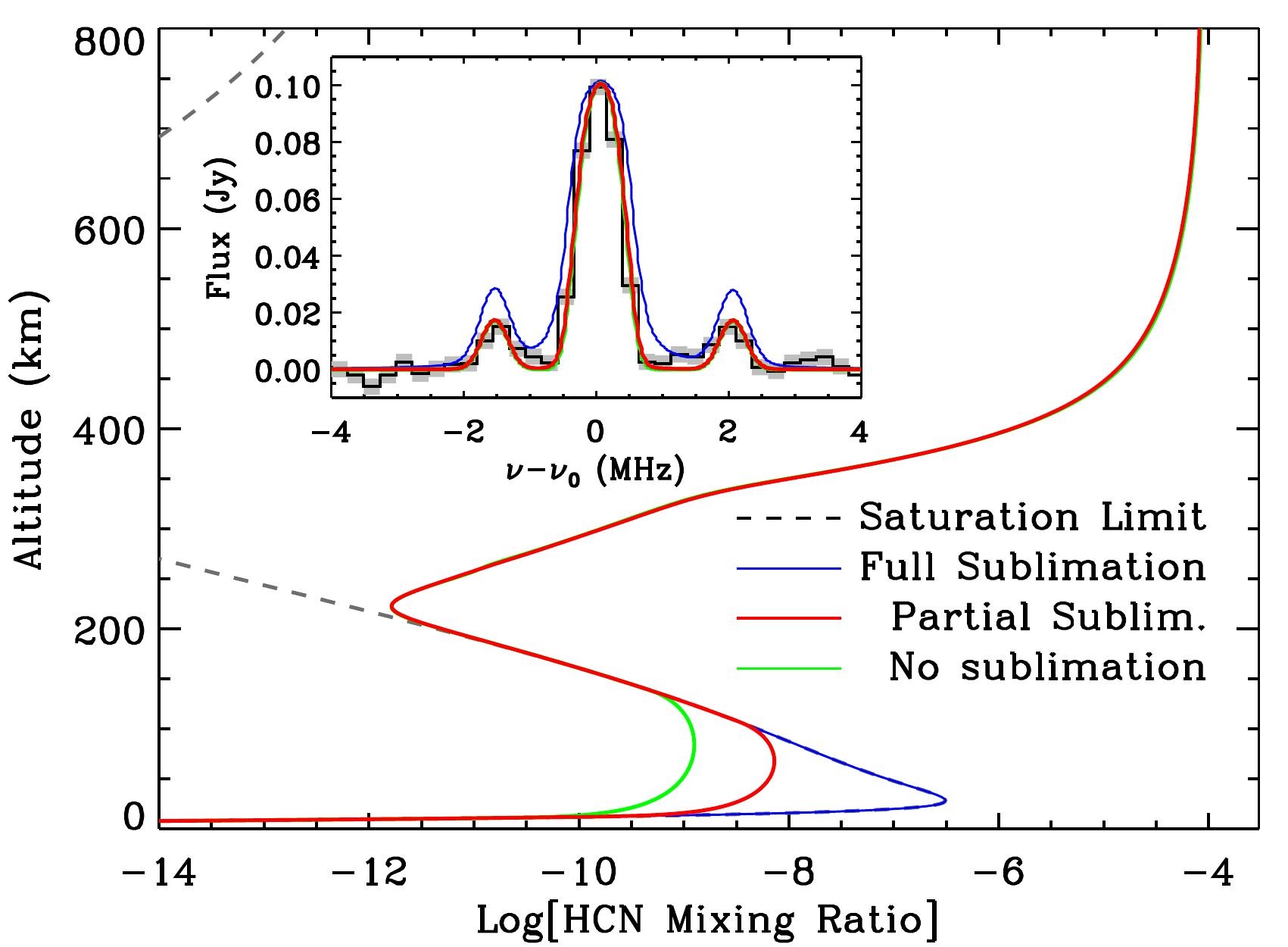




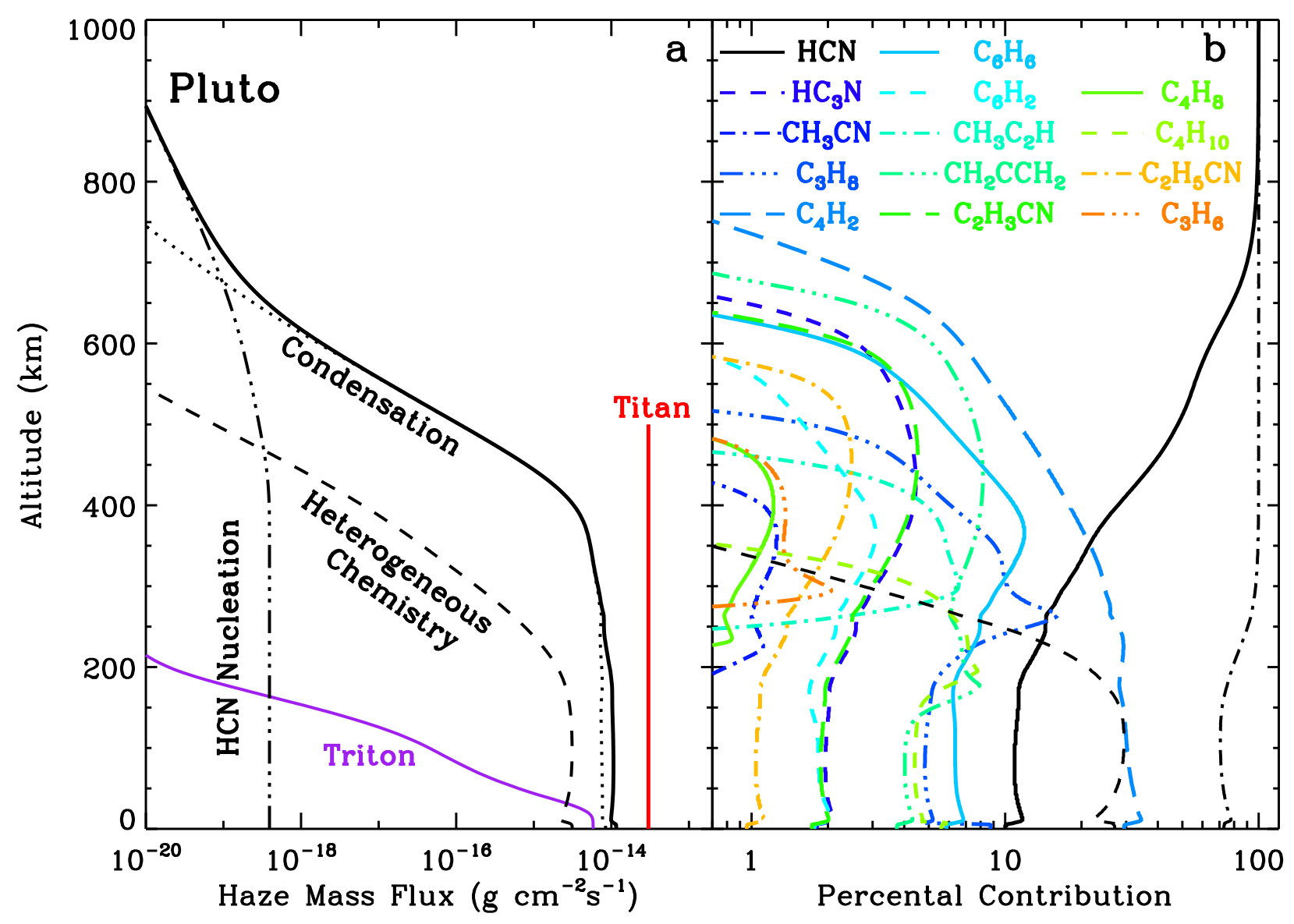


$\log [d M($ Coag. $) /(d M($ Cond. $)+d M($ Coag. $))]$

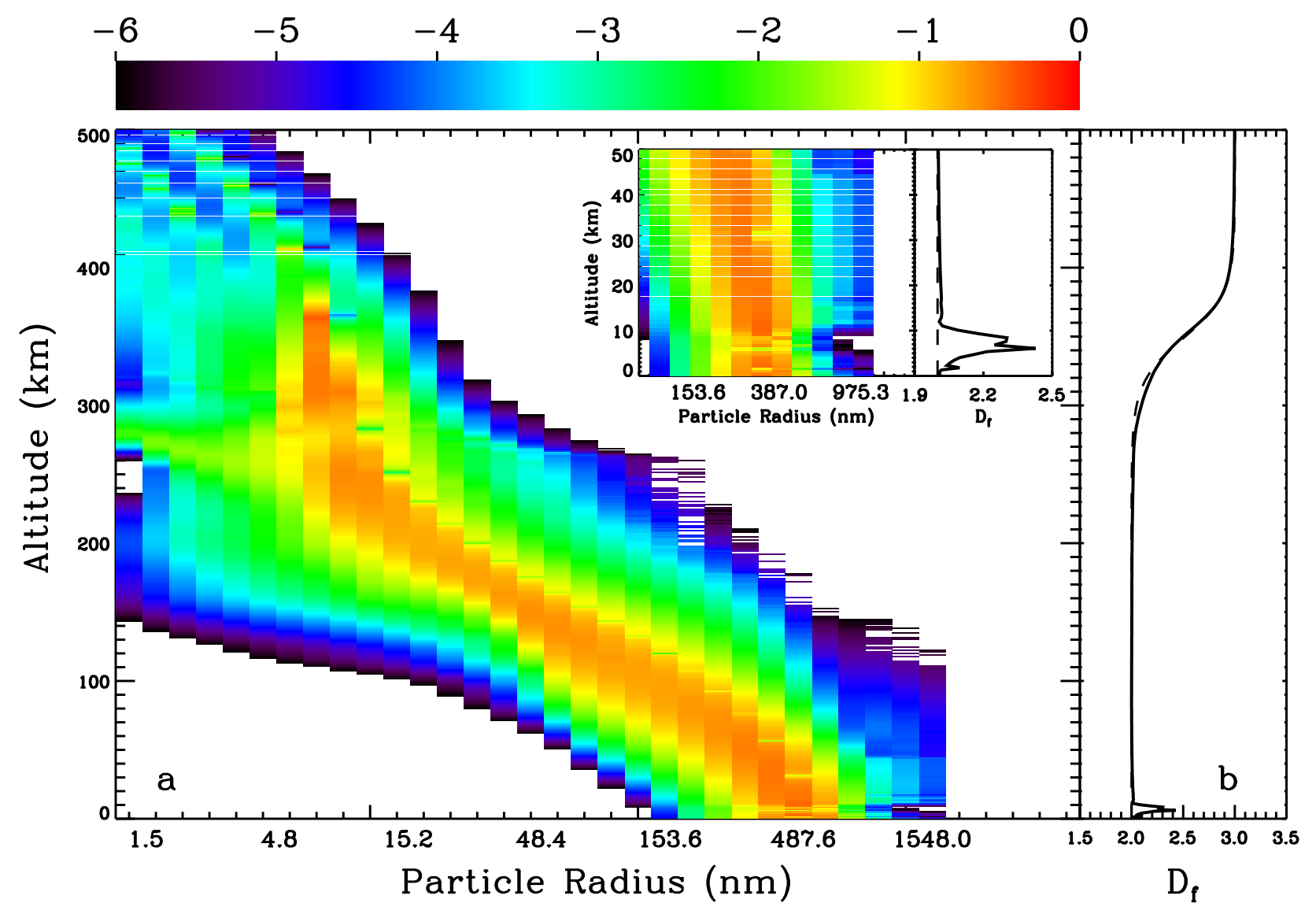




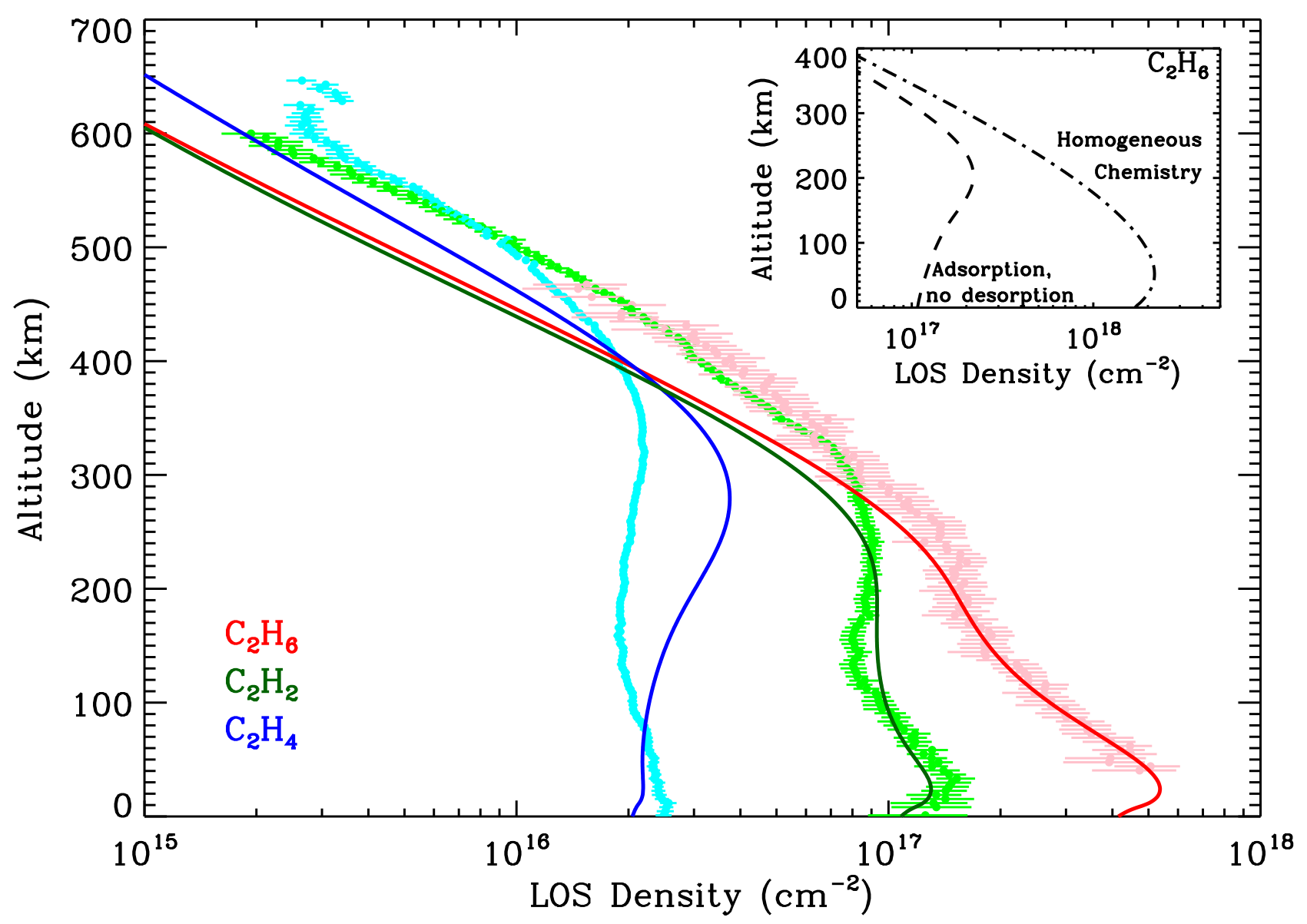



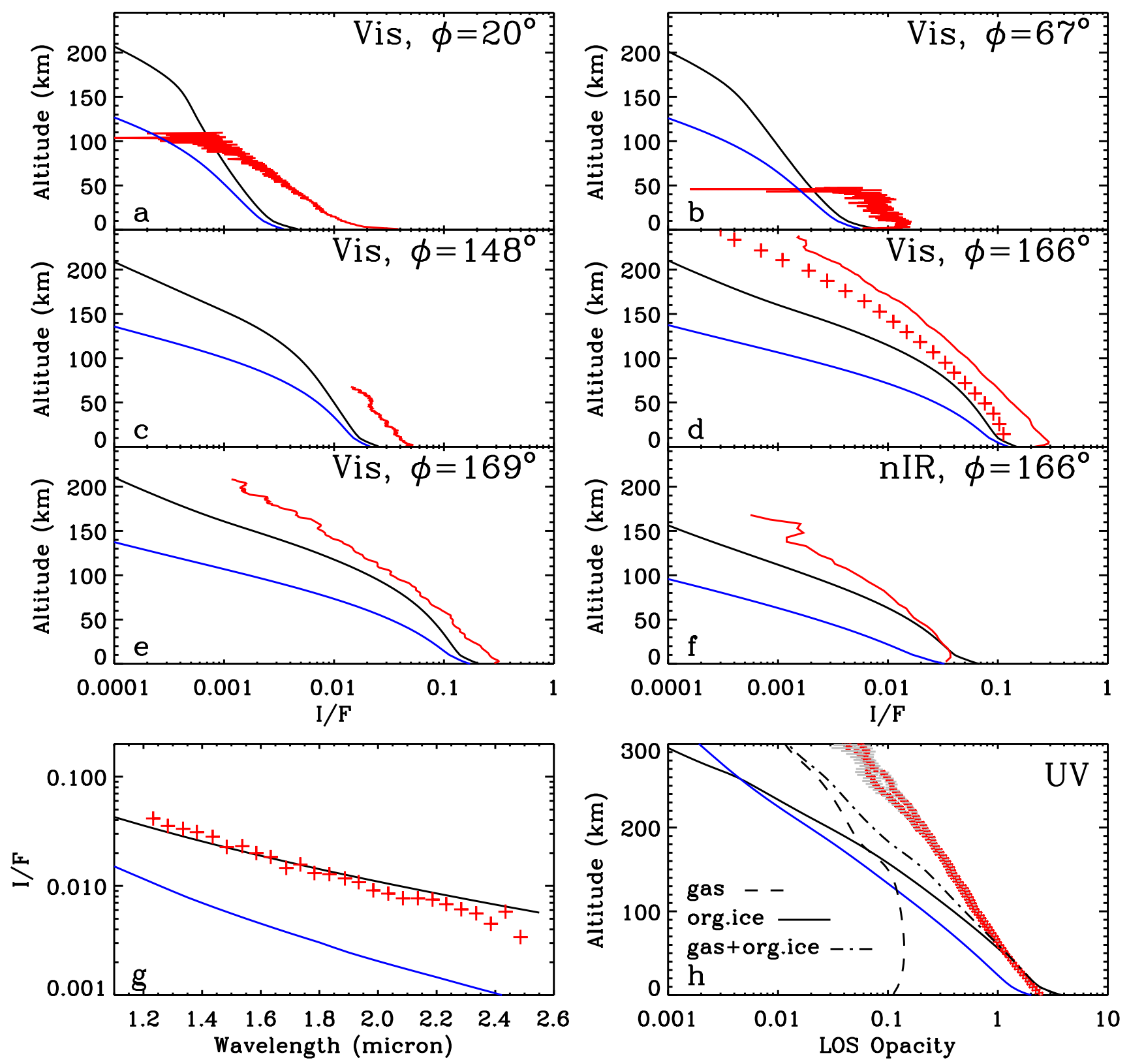


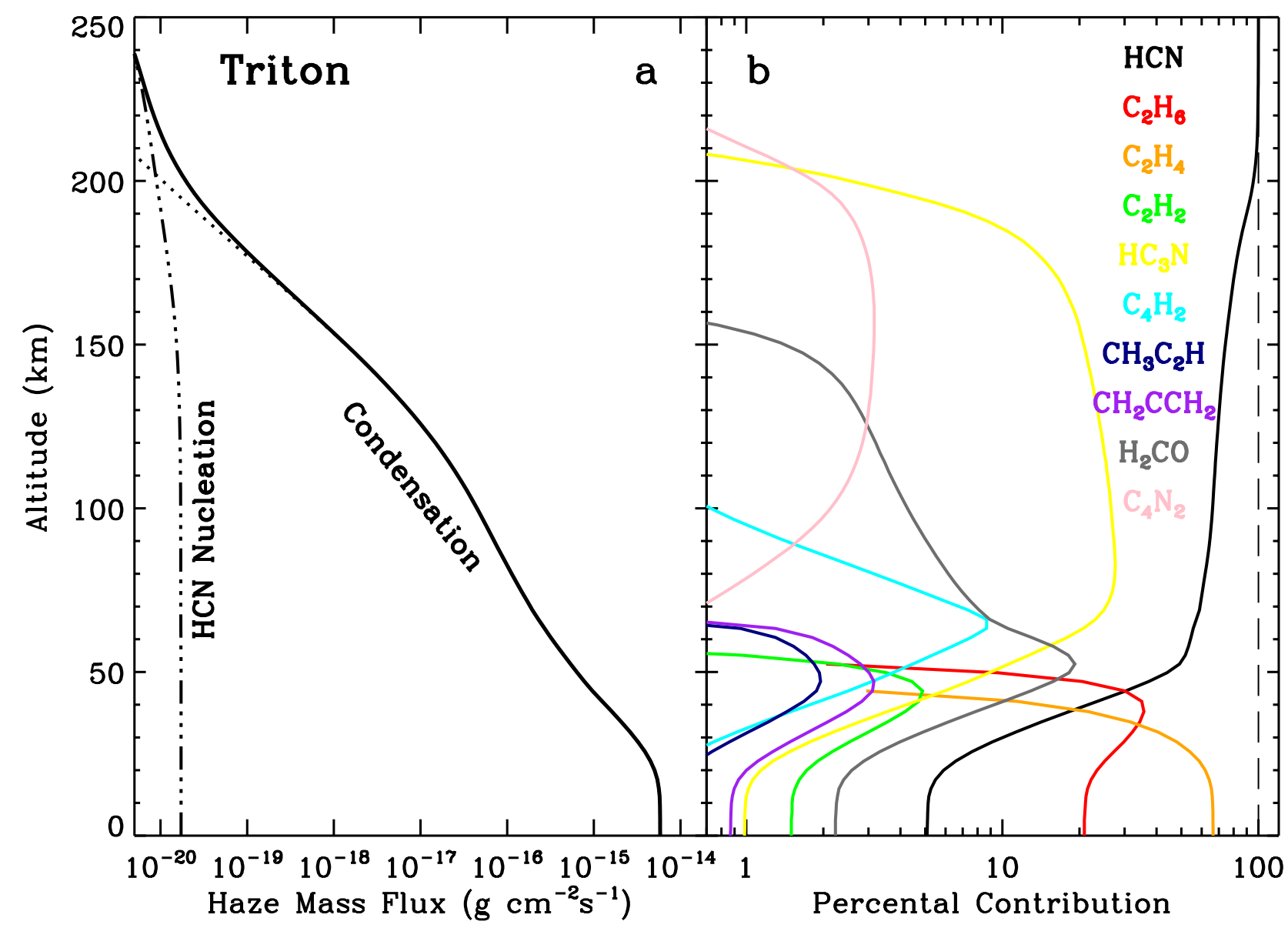

$\log [\mathrm{dM}($ Coag. $) /(\mathrm{dM}$ (Cond.) $+\mathrm{dM}($ Coag. $))]$

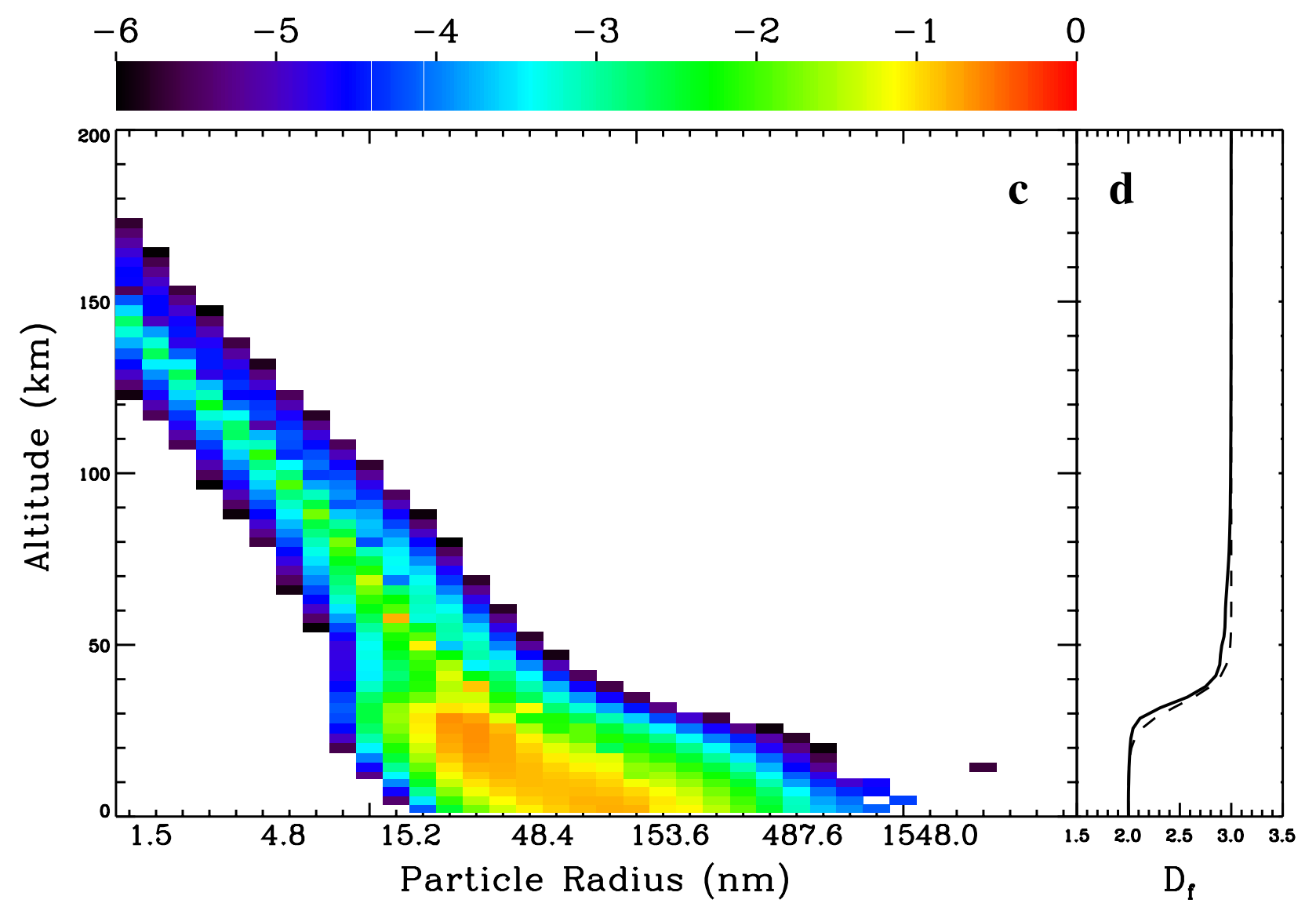




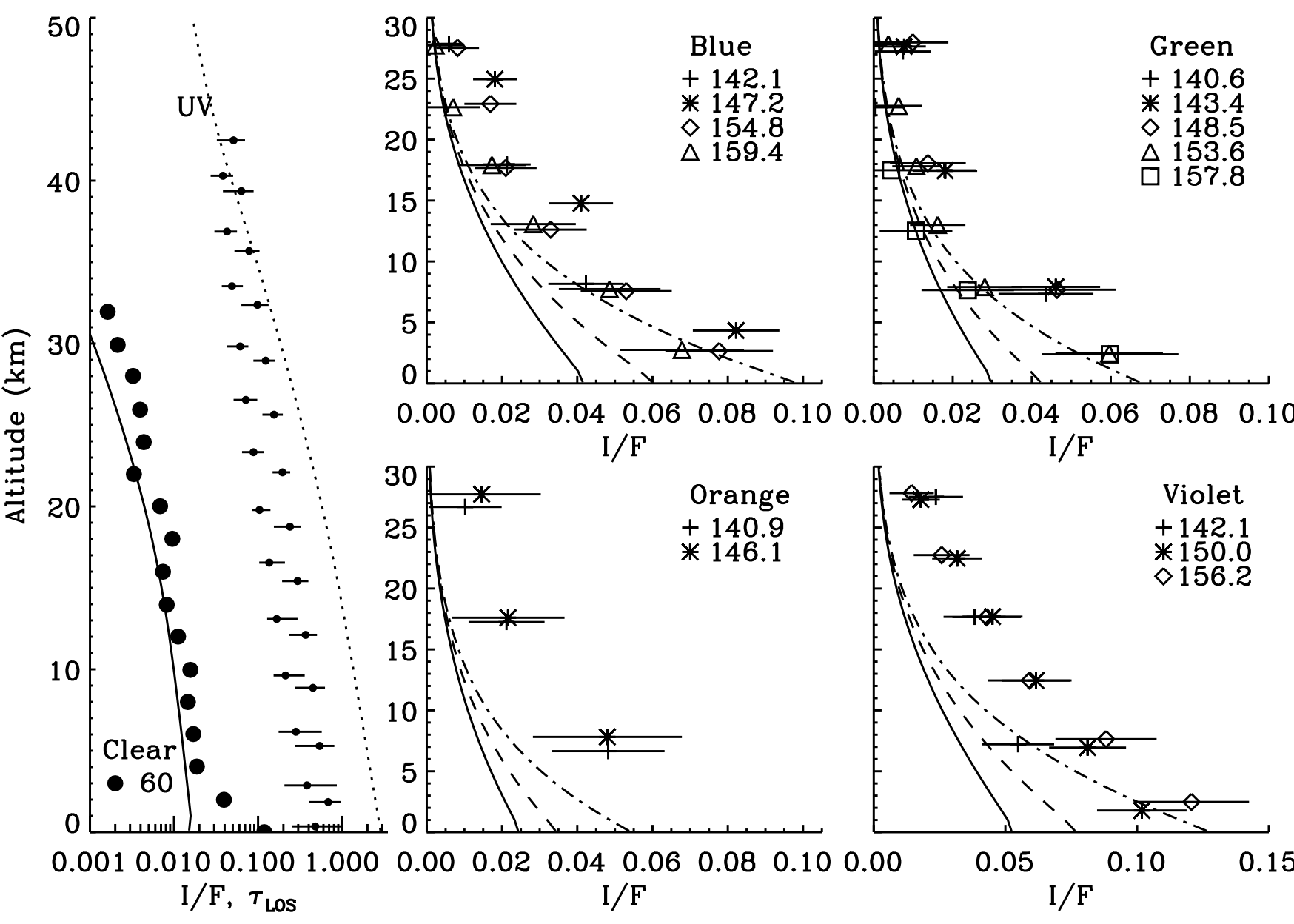




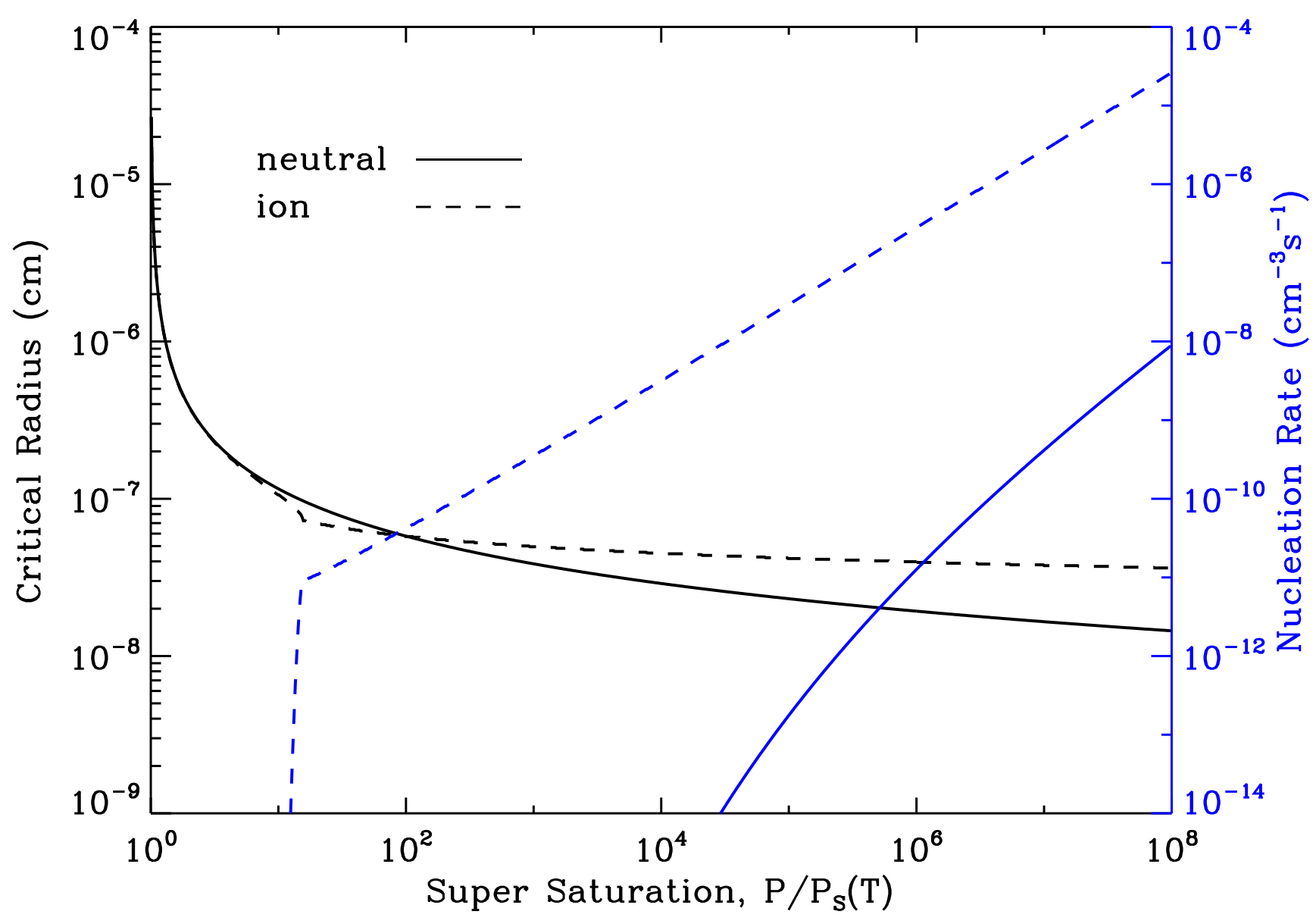




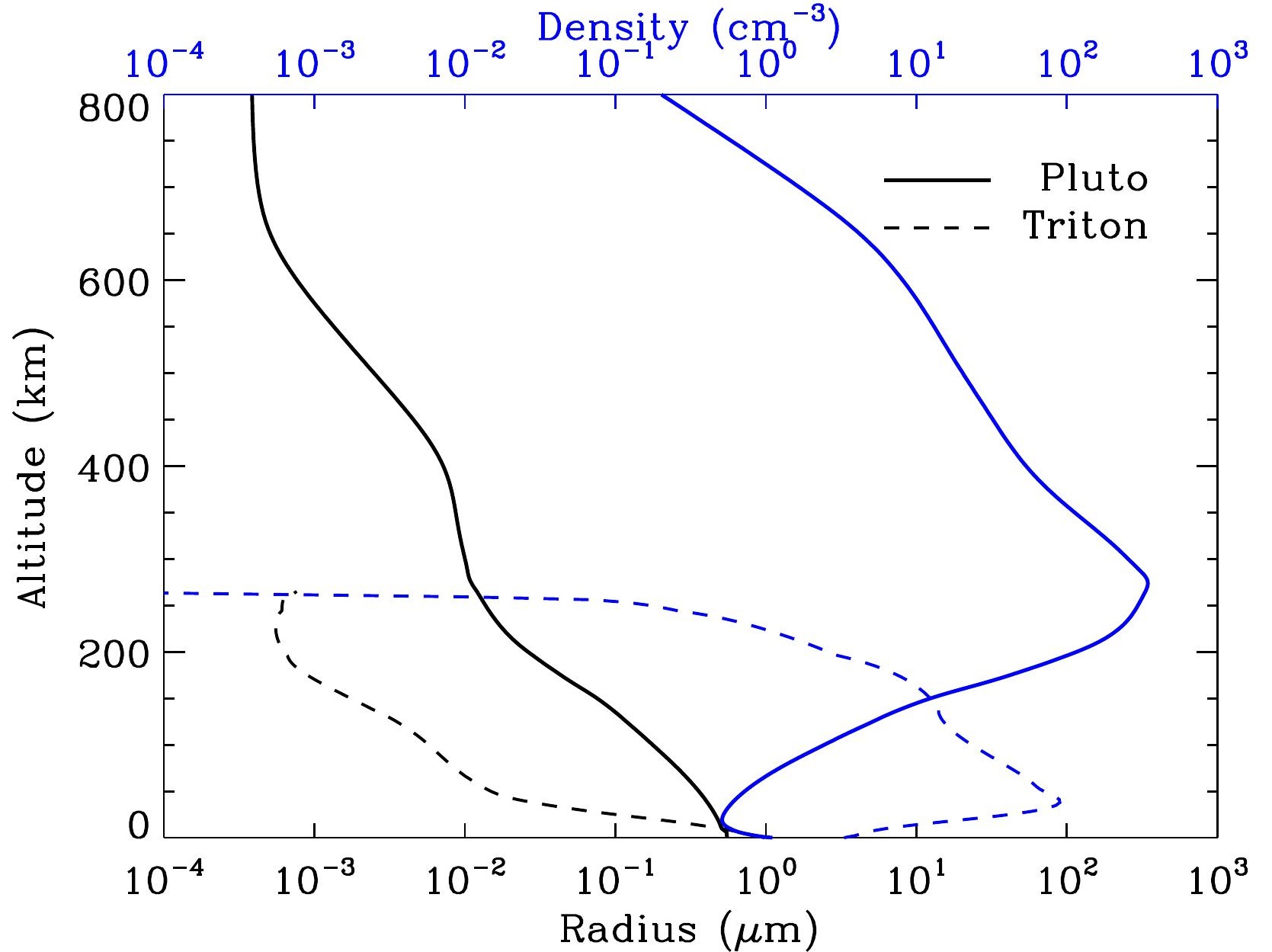




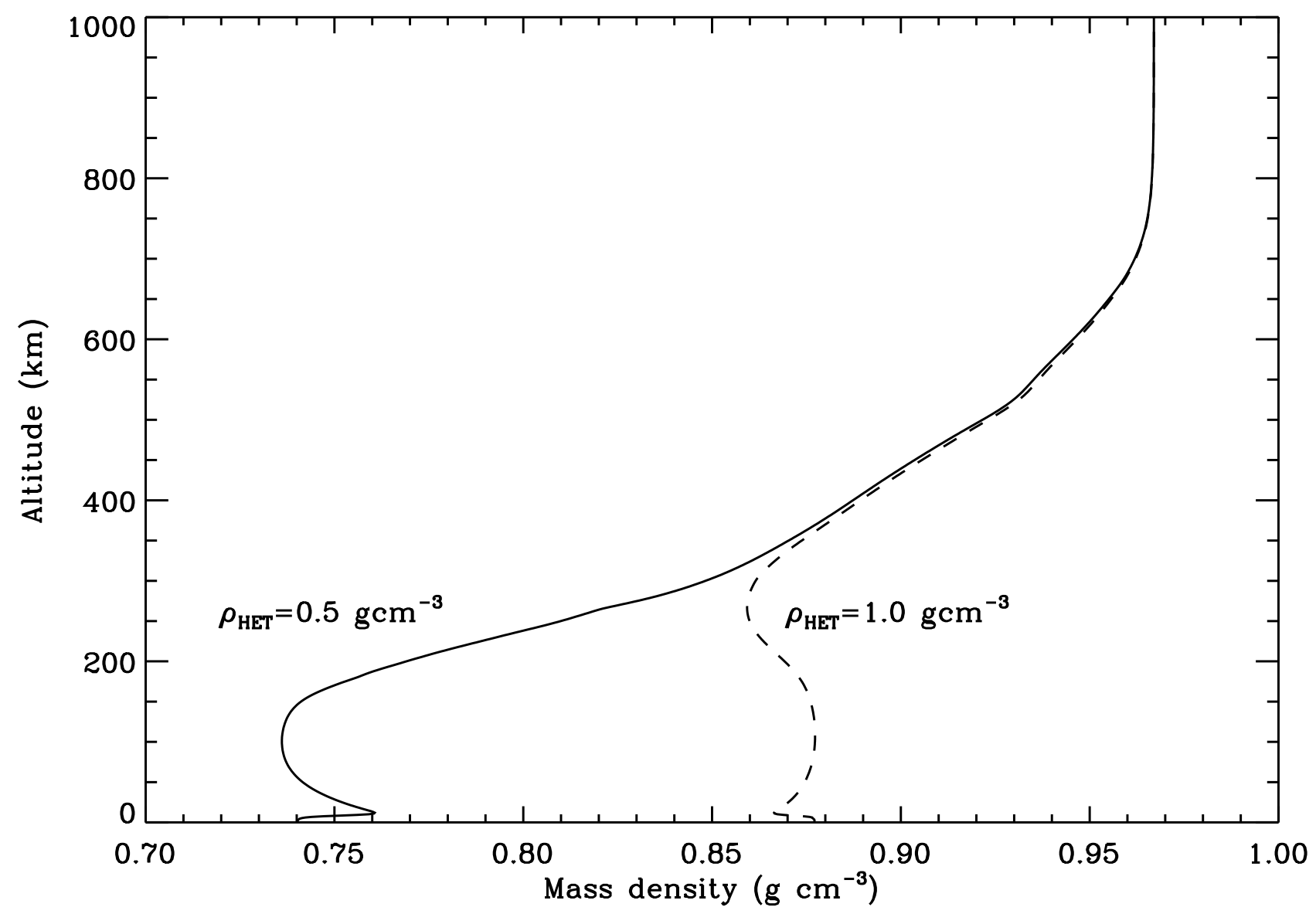




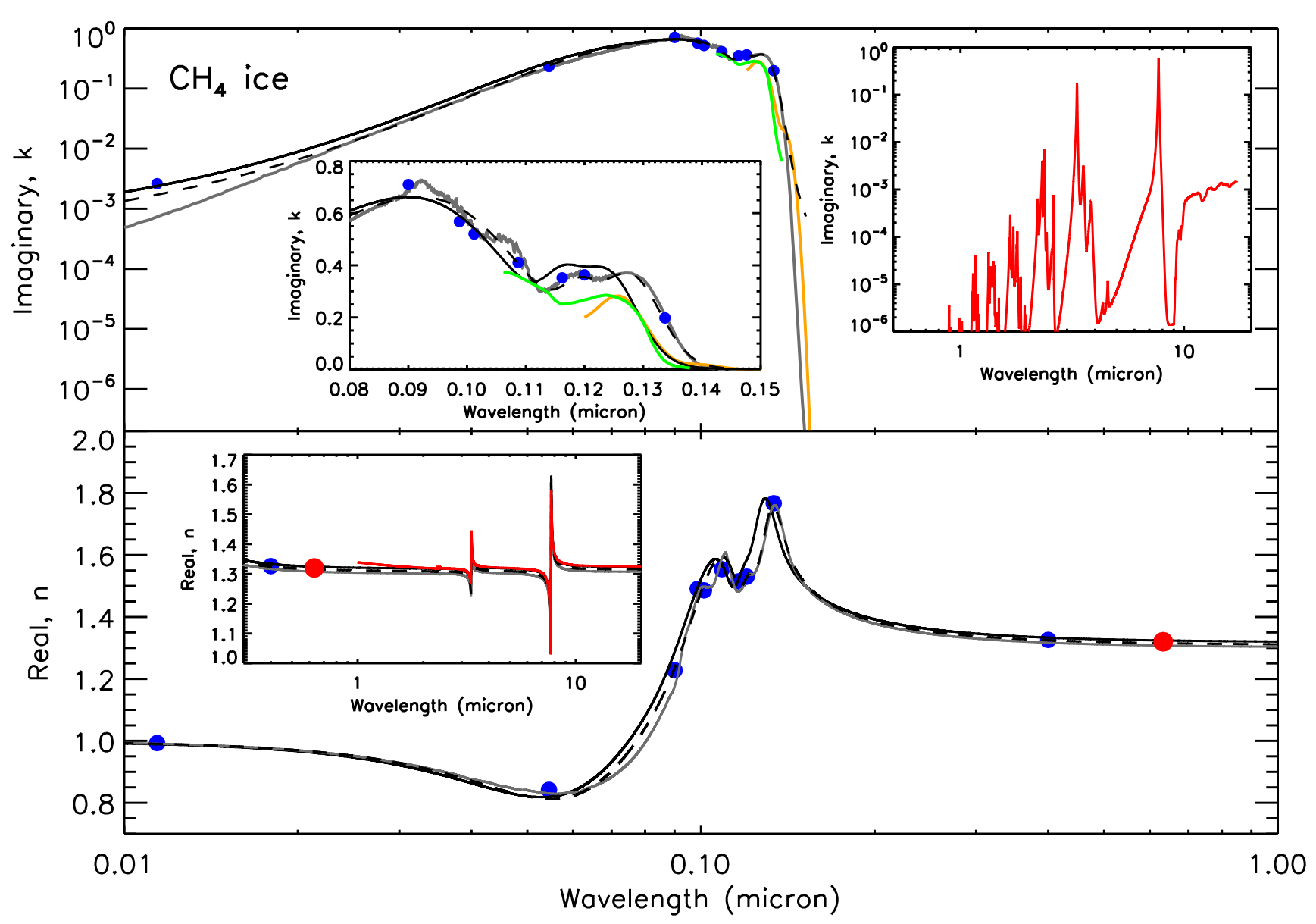




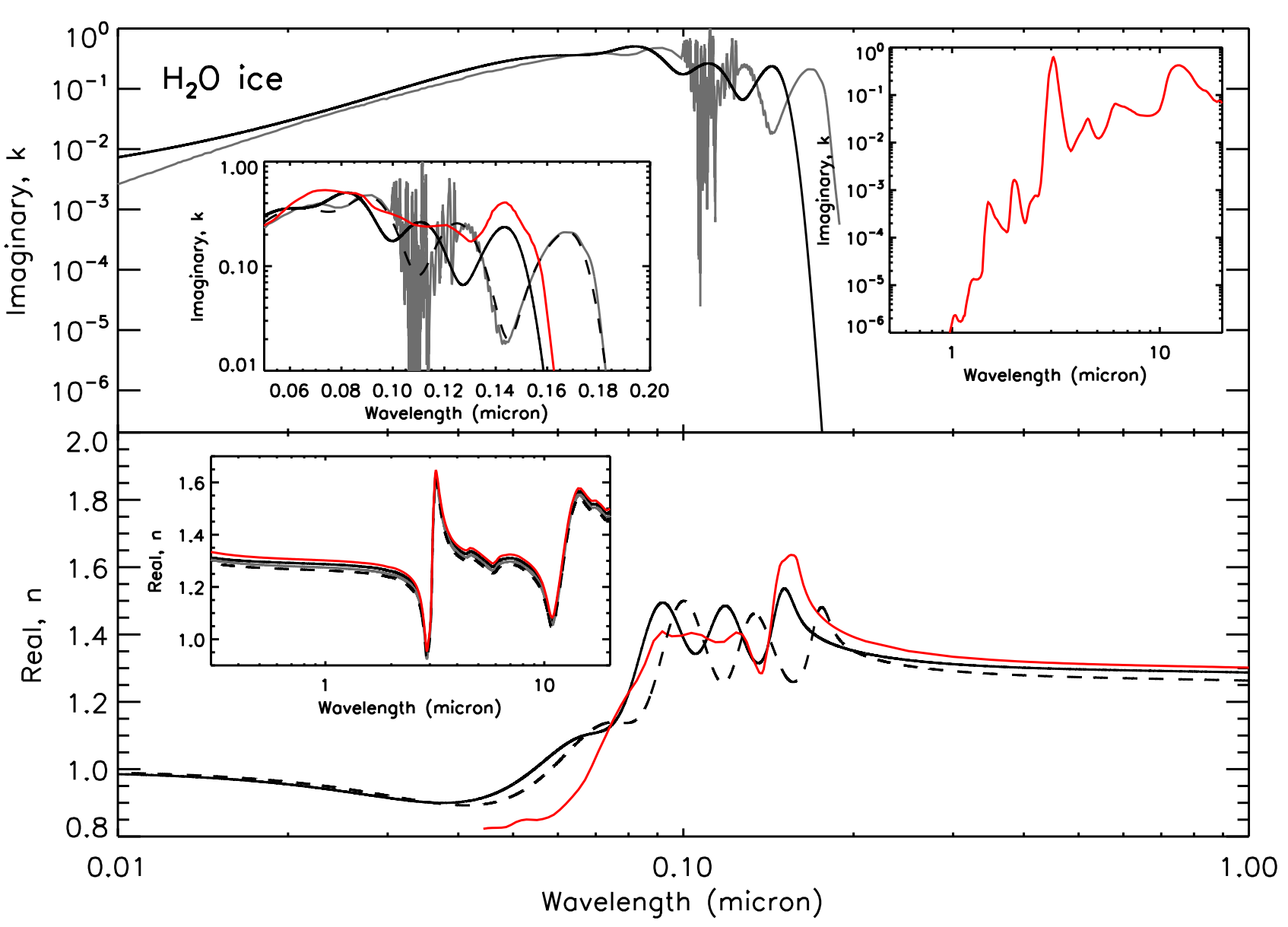




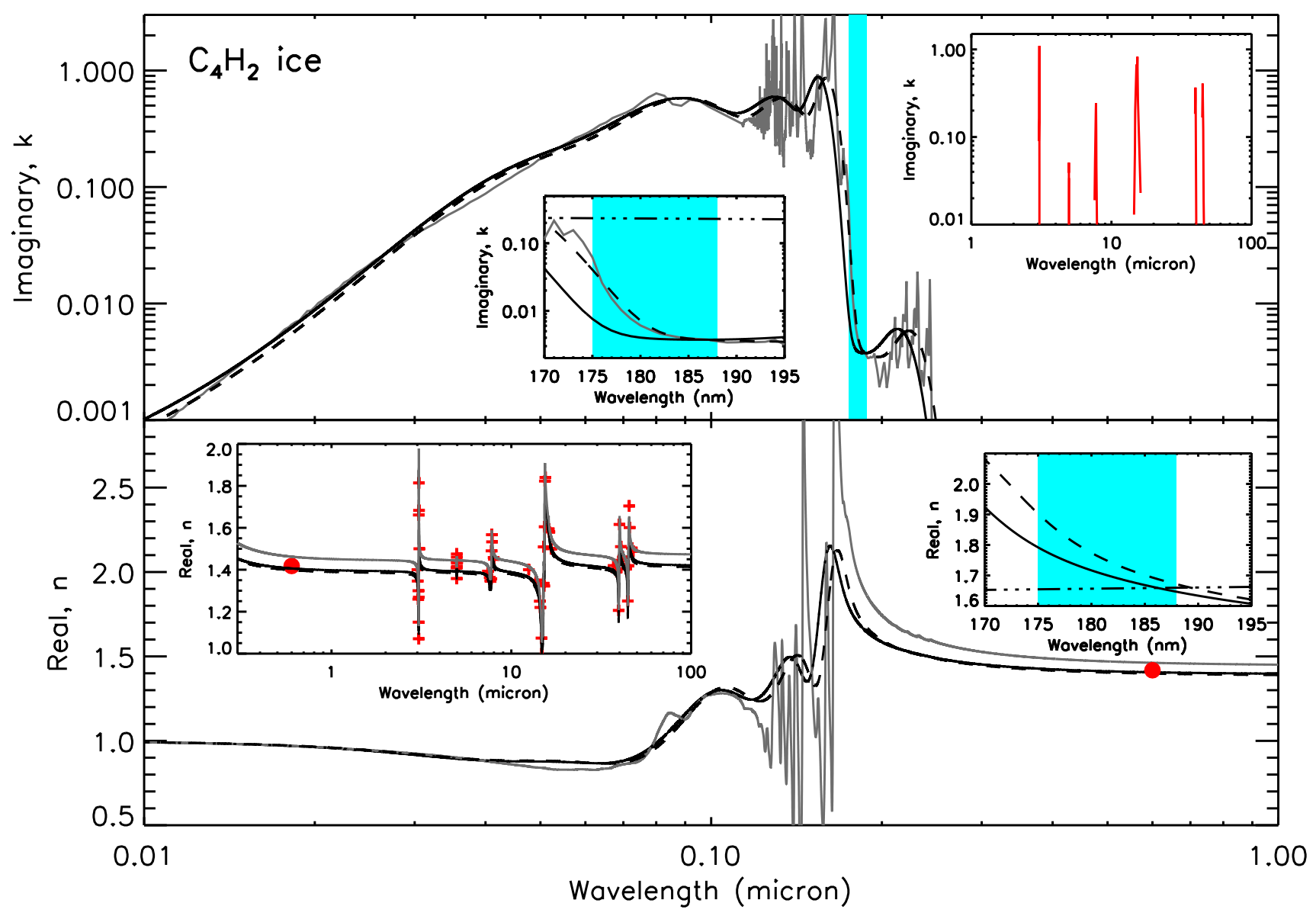




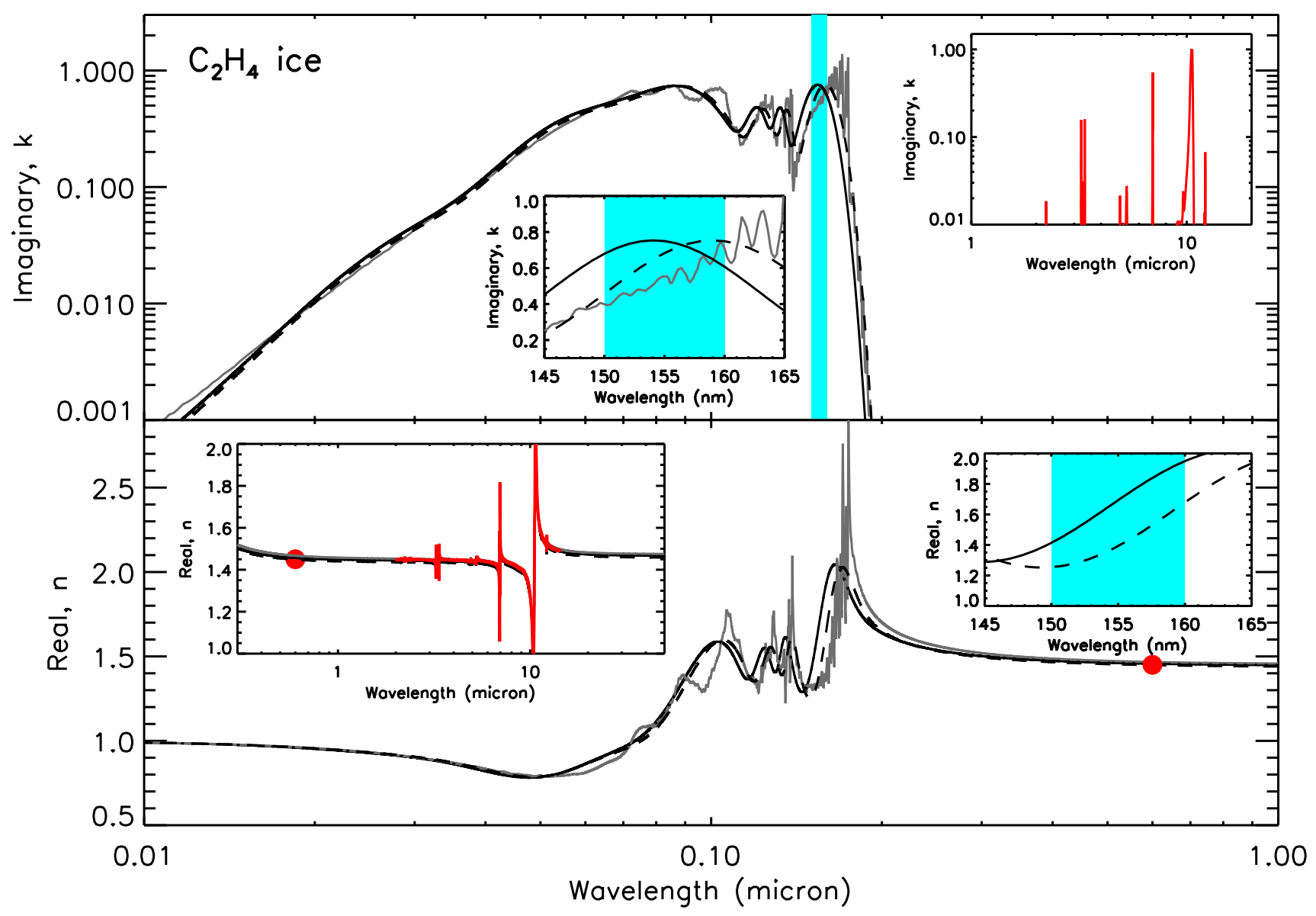

